Reduced version in Proceedings of the FEA 2004 Conference at MIT, Cambridge, Massachusetts, November 8-10, and in: Pykhtin, M. (Editor), Counterparty Credit Risk Modeling: Risk Management, Pricing and Regulation. Risk Books, 2005, London.

\title{
Credit Default Swap Calibration and Equity Swap Valuation under Counterparty Risk with a Tractable Structural Model
}

\author{
Damiano Brigo Marco Tarenghi \\ Credit Models \\ Banca IMI \\ Corso Matteotti 6 \\ 20121 Milano, Italy \\ Posted on SSRN.com on August 24, 2004 \\ http://ssrn.com/abstract=581302 \\ http://www.damianobrigo.it
}

First version: March 1, 2004. This Version: March 8, 2005

\begin{abstract}
In this paper we develop a tractable structural model with analytical default probabilities depending on some dynamics parameters, and we show how to calibrate the model using a chosen number of Credit Default Swap (CDS) market quotes. We essentially show how to use structural models with a calibration capability that is typical of the much more tractable credit-spread based intensity models. We apply the structural model to a concrete calibration case and observe what happens to the calibrated dynamics when the CDS-implied credit quality deteriorates as the firm approaches default. Finally we provide a typical example of a case where the calibrated structural model can be used for credit pricing in a much more convenient way than a calibrated reduced form model: The pricing of counterparty risk in an equity swap.
\end{abstract}

\section{Keywords}

Credit Derivatives, Structural Models, Black Cox Model, Credit Default Swaps, Calibration, Analytical Tractability, Monte Carlo Simulation, Equity Swaps, Counterparty Risk, Barrier Options. 


\section{Contents}

1 Introduction 3

2 The Black Cox approach and Barrier options formulas 4

3 Calibration of the structural model to CDS data 7

3.1 First Numerical Example . . . . . . . . . . . . . . 12

3.2 Finding $H$ : Credit spread method and protection/excursion analogy method ........................ 15

4 Further Model Specification and a Case Study: the Parmalat Crisis 16

5 A fundamental example: Pricing Counterparty Risk in Equity Swaps 22

6 Conclusions 29

7 Appendix: Results of the case study calibrations and market data 30

7.1 Credit spread based barrier parameter $H \ldots \ldots$. . . . . . . 30

7.2 Excursion/protection analogy barrier parameter $H \ldots \ldots$. . . . . . . 32

7.3 Discount Curves . . . . . . . . . . . . . . . . 34 


\section{Introduction}

Modelling firms default is an important issue, especially in recent times where the market is experiencing an increasing interest in credit derivatives trading. Pricing models can be divided into two main categories: (i) reduced form models and (ii) structural models.

Reduced form models (also called intensity models when a suitable context is possible) describe default by means of an exogenous jump process; more precisely, the default time is the first jump time of a Poisson process with deterministic or stochastic (Cox process) intensity. Here default is not triggered by basic market observables but has an exogenous component that is independent of all the default free market information. Monitoring the default free market does not give complete information on the default process, and there is no economic rationale behind default. This family of models is particularly suited to model credit spreads and in its basic formulation is easy to calibrate to Credit Default Swap (CDS) data. See references at the beginning of Chapter 8 in Bielecki and Rutkowski (2001) for a summary of the literature on intensity models. We cite here Duffie and Singleton (1999) and Lando (1998), and Brigo and Alfonsi (2003) as a reference for explicit calibration of a tractable stochastic intensity model to CDS data.

Structural models are based on the work by Merton (1974), in which a firm life is linked to its ability to pay back its debt. Let us suppose that a firm issues a bond to finance its activities and also that this bond has maturity $T$. At final time $T$, if the firm is not able to reimburse all the bondholders we can say that there has been a default event. In this context default may occur only at final time $T$ and is triggered by the value of the firm being below the debt level. In a more realistic and sophisticated structural model (Black and Cox (BC) (1976), part of the family of first passage time models) default can happen also before maturity $T$. In first passage time models the default time is the first instant where the firm value hits from above either a deterministic (possibly time varying) or a stochastic barrier, ideally associated with safety covenants forcing the firm to early bankruptcy in case of important credit deterioration. In this sense the firm value is seen as a generic asset and these models use the same mathematics of barrier options pricing models. For a summary of the literature on structural models, possibly with stochastic interest rates and default barriers, we refer for example to Chapter 3 of Bielecki and Rutkowski (2001). It is important to notice that structural models make some implicit but important assumptions: They assume that the firm value follows a random process similar to the one used to describe generic stocks in equity markets, and that it is possible to observe this value at any time. Therefore, unlike intensity models, here the default process can be completely monitored based on default free market information and comes less as a surprise. However, structural models in their basic formulations and with standard barriers (Merton, BC) have few parameters in their dynamics and cannot be calibrated exactly to structured data such as CDS quotes along different maturities.

In this paper we plan to effectively use a structural model in the ideal "territory" of intensity models, i.e. to describe default probabilities in a way that is rich enough to calibrate CDS quotes. First of all, in Section 2 we show how standard barrier options formulas can be used to find these probabilities, and in particular we investigate 
the case of time dependent parameters in the dynamics of the underlying process. In Section 3 we show how to calibrate the parameters of the model to market prices of single name CDS's (in a way similar to the procedure used to calibrate intensities in reduced form models). In Section 4 we consider the concrete case of a firm approaching default, and we show how our proposed structural model calibration changes as the company credit quality (as summarized by its CDS quotes) deteriorates in time. The calibrated model is useful for subsequent pricing of more sophisticated derivatives depending on default. Indeed, in Section 5 we see an important example of how our structural model can be used to price some products involving considerations from both the credit and the equity market. The product we consider is an equity return swap with counterparty risk. In this context we have to take care of the correlation between the counterparty and the underlying, and this is done much more conveniently in structural models than in intensity models.

Finally, we have considered tractable extensions of the AT1P model based on introducing scenarios on the value of the firm volatility and safety barrier. This framework allows to consider situations where the balance sheets information can be uncertain or hid some developments. This work and the related SVBAT1P models are reported in Brigo and Tarenghi (2005), with a calibration case study on CDS data.

\section{The Black Cox approach and Barrier options formulas}

The fundamental hypothesis of the model we resume here is that the underlying process is a Geometric Brownian Motion (GBM), which is also the kind of process commonly used for equity stocks in the Black Scholes model.

Classical structural models (Merton, Black Cox) postulate a GBM (Black and Scholes) lognormal dynamics for the value of the firm $V$. This lognormality assumption is considered to be acceptable. Crouhy et al (2000) report that "this assumption [lognormal V] is quite robust and, according to KMVs own empirical studies, actual data conform quite well to this hypothesis.".

In these models the value of the firm $V$ is the sum of the firm equity value $S$ and of the firm debt value $D$. The firm equity value $S$, in particular, can be seen as a kind of (vanilla or barrier-like) option on the value of the firm $V$. Merton typically assumes a zero-coupon debt at a terminal maturity $T$. Black Cox assume, besides a possible zero coupon debt, safety covenants forcing the firm to declare bankruptcy and pay back its debt with what is left as soon as the value of the firm itself goes below a "safety level" barrier. This is what introduces the barrier option technology in structural models for default.

More in detail, in Merton's model there is a debt maturity $\bar{T}$, a debt face value $L$

and the company defaults at final maturity (and only then) if the value of the firm $V_{\bar{T}}$ is below the debt $L$ to be paid.

The debt value at time $t<\bar{T}$ is thus

$$
D_{t}=\mathbb{E}_{t}\left[D(t, \bar{T}) \min \left(V_{\bar{T}}, L\right)\right]=\mathbb{E}_{t}\left[D(t, \bar{T})\left[V_{\bar{T}}-\left(V_{\bar{T}}-L\right)^{+}\right]\right]=
$$




$$
=\mathbb{E}_{t}\left[D(t, \bar{T})\left[L-\left(L-V_{\bar{T}}\right)^{+}\right]\right]=P(t, \bar{T}) L-\operatorname{Put}\left(t, \bar{T} ; V_{t}, L\right)
$$

where Put(time, maturity, underlying, strike) is a put option price, and the stochastic discount factor at time $t$ for maturity $T$ is denoted by $D(t, T)=B(t) / B(T)$, where $B(t)=\exp \left(\int_{0}^{t} r_{u} d u\right)$ denotes the bank-account numeraire, $r$ being the instantaneous short interest rate. Since we will assume deterministic interest rates, in our case $D(t, T)=P(t, T)$, the zero coupon bond price at time $t$ for maturity $T$.

The equity value can be derived as a difference between the value of the firm and the debt:

$$
S_{t}=V_{t}-D_{t}=V_{t}-P(t, \bar{T}) L+\operatorname{Put}\left(t, \bar{T} ; V_{t}, L\right)=\operatorname{Call}\left(t, \bar{T} ; V_{t}, L\right)
$$

so that, as is well known, in Merton's model the equity can be interpreted as a call option on the value of the firm.

Let us now move to the Black Cox (BC) model. In this model we have safety covenants in place, in that the firm is forced to reimburse its debt as soon as its value $V_{t}$ hits a low enough "safety level" $\widehat{H}(t)$. The choice of this safety level is not easy. Assuming a debt face value of $L$ at final maturity $\bar{T}$ as before, an obvious candidate for this "safety level" is the final debt present value discounted back at time $t$, i.e. $L P(t, \bar{T})$. However, one may want to cut some slack to the counterparty, giving it some time to recover even if the level goes below $L P(t, \bar{T})$, and the "safety level" can be chosen to be lower than $L P(t, \bar{T})$.

We will come back to this issue later on. For the time being we just assume $V$ to be a geometric Brownian motion.

$$
d V_{t}^{*}=V_{t}^{*} \mu_{t}^{*} d t+V_{t}^{*} \sigma_{t} d W(t), \quad V_{0}^{*}=V_{0}
$$

and impose a time dependent safety barrier $H^{*}(t)$. For the time being let us assume to have constant parameters (i.e. $\mu_{t}^{*}=\mu^{*}$ and $\sigma_{t}=\sigma$ ) and a constant barrier $\left(H^{*}(t)=H^{*}\right)$. We set ourselves in the risk neutral measure, so that the drift term has the standard form of interest rate minus a payout ratio (i.e. $\mu^{*}=r-q^{*}$ ) and (11) is a standard Geometric Brownian Motion. Let us pretend for a moment that we are just doing derivatives pricing in an equity market with underlying $V$. In the literature it is possible to find many analytical formulas for barrier options pricing such as, for example, knock-in or knock-out options, and also digital options. One particular case is the down and out digital option or down and out bond (DOB), that is a contract paying one unit of currency at maturity $T$ if, between the starting date of the contract and its maturity $T$, the underlying never touches the barrier $H^{*}$ $\left(H^{*}<V_{0}^{*}\right)$ from above. If we go back to the default interpretation with $H^{*}$ as safety barrier level and $T<\bar{T}$, this option price is actually the price of a defaultable zero coupon bond with no recovery in the structural model framework. Now, if we call $\tau$ the first time instant where the process hits the barrier from above, the price of this option in the risk-neutral framework is given by

$$
\operatorname{DOB}(0, T)=\mathbb{E}\left\{D(0, T) \mathbf{1}_{\{\tau>T\}}\right\}
$$

where $\mathbb{E}$ is the risk neutral expectation associated with the risk neutral measure $\mathbb{Q}$. Under our deterministic rates framework (or under interest rates that are independent of the underlying process) we can write

$$
\operatorname{DOB}(0, T)=P(0, T) \mathbb{E}\left\{\mathbf{1}_{\{\tau>T\}}\right\}=P(0, T) \mathbb{Q}\{\tau>T\} .
$$


The last factor is the (risk-neutral) probability of never touching the barrier before $T$, also called survival probability. By means of stochastic calculus it is possible to derive analytically the price of the option by explicitly computing (3) (see for example Bielecki and Rutkowski (2001)):

$$
\operatorname{DOB}(0, T)=P(0, T)\left[\Phi\left(d_{1}\right)-\left(\frac{V_{0}}{H^{*}}\right)^{1-2\left(r-q^{*}\right) /\left(\sigma^{2}\right)} \Phi\left(d_{2}\right)\right]
$$

with $d_{1,2}=\left( \pm \log \frac{V_{0}}{H^{*}}+\left(r-q^{*}-\frac{\sigma^{2}}{2}\right) T\right) /(\sigma \sqrt{T})$. By comparing equations (3i) and (44) we obtain

$$
\mathbb{Q}\{\tau>T\}=\left[\Phi\left(d_{1}\right)-\left(\frac{V_{0}}{H^{*}}\right)^{1-2\left(r-q^{*}\right) /\left(\sigma^{2}\right)} \Phi\left(d_{2}\right)\right] .
$$

Similar formulas can be obtained also for a barrier that is not necessarily constant in time (or "flat"), but with a particular exponential shape (see again Bielecki and Rutkowski (2001) for more details).

When relaxing the assumption of constant parameters in the $V^{*}$ dynamics, the situation becomes much more complicated. In this case, even with a flat barrier, it is not possible to find closed form pricing formulas. However, some recent work dealing with option pricing on underlying assets having time dependent parameters in the dynamics shows that it is possible to find analytical barrier option prices when the barrier has a particular curved shape depending partly on the dynamics parameters. See for example Rapisarda (2003), who builds on the fundamental work of Lo et al. (2003), where the formulas are expressed in a shape resembling the classical constant coefficients Black-Scholes formulas. Again, let us assume an underlying process like (1), with $\mu_{t}^{*}=r_{t}-q_{t}^{*}$, where $r$ and $q^{*}$ are respectively the time varying instantaneous risk free interest rate and payout ratio, and consider an option with maturity $T$. Let us take a barrier of the form:

$$
H^{*}(t)=H \exp \left(-\int_{t}^{T}\left(r_{s}-q_{s}^{*}-(1+2 \beta) \frac{\sigma_{s}^{2}}{2}\right) d s\right)
$$

depending on a parameter $\beta$ and on the constant reference value $H$. For this special barrier it is possible to obtain exact formulas for many different barrier options when the underlying is $V^{*}$. Obviously, if we had to price an option with a given constant barrier, the price obtained with these formulas would not be properly correct, but it would be a good approximation if we chose the barrier profile $H^{*}(t)$ as close as possible to the given flat barrier value. It can be shown that the value of $\beta$ keeping the barrier as flat as possible is given by

$$
\beta^{*}=\frac{\int_{0}^{T}\left(\int_{t}^{T}\left(r_{s}-q_{s}^{*}-\frac{\sigma_{s}^{2}}{2}\right) d s\right)\left(\int_{t}^{T} \sigma_{s}^{2} d s\right) d t}{\int_{0}^{T}\left(\int_{t}^{T} \sigma_{s}^{2} d s\right)^{2} d t}
$$

and this reduces to $\beta^{*}=\left(r-q^{*}-\sigma^{2} / 2\right) /\left(\sigma^{2}\right)$ in the case of constant coefficients (where, as expected, $H^{*}(t)=H$ ). 
Let us go back to the time-varying barrier (6). In this framework

$\operatorname{DOB}(0, T)=P(0, T) \cdot\left[\Phi\left(\frac{\log \frac{V_{0}}{H}+\int_{0}^{T} v_{s} d s}{\sqrt{\int_{0}^{T} \sigma_{s}^{2} d s}}\right)-\left(\frac{H^{*}(0)}{V_{0}}\right)^{2 \beta} \Phi\left(\frac{\log \frac{H^{*}(0)^{2}}{V_{0} H}+\int_{0}^{T} v_{s} d s}{\sqrt{\int_{0}^{T} \sigma_{s}^{2} d s}}\right)\right]$

where $v_{t}=r_{t}-q_{t}^{*}-\frac{\sigma_{t}^{2}}{2}$ and, using again (3), we find

$$
\mathbb{Q}\{\tau>T\}=\left[\Phi\left(\frac{\log \frac{V_{0}}{H}+\int_{0}^{T} v_{s} d s}{\sqrt{\int_{0}^{T} \sigma_{s}^{2} d s}}\right)-\left(\frac{H^{*}(0)}{V_{0}}\right)^{2 \beta} \Phi\left(\frac{\log \frac{H^{*}(0)^{2}}{V_{0} H}+\int_{0}^{T} v_{s} d s}{\sqrt{\int_{0}^{T} \sigma_{s}^{2} d s}}\right)\right]
$$

where we recall that $H^{*}(0)=H \exp \left(-\int_{0}^{T}\left(r_{s}-q_{s}^{*}-(1+2 \beta) \frac{\sigma_{s}^{2}}{2}\right) d s\right)$.

In the next section we show how this formula can be used to calibrate a given structural model to market data, in particular to CDS's quotes.

\section{Calibration of the structural model to CDS data}

Since we are dealing with default probabilities of firms, it is straightforward to think of financial instruments depending on these probabilities and whose final aim is to protect against the default event. One of the most representative protection instruments is the Credit Default Swap (CDS). CDS's are contracts that have been designed to offer protection against default. Consider two companies "A" (the protection buyer) and "B" (the protection seller) who agree on the following.

If a third reference company "C" (the reference credit) defaults at a time $\tau_{C} \in$ $\left(T_{a}, T_{b}\right]$, "B" pays to "A" at time $\tau=\tau_{C}$ itself a certain "protection" cash amount LGD (Loss Given the Default of "C"), supposed to be deterministic in the present paper. This cash amount is a protection for "A" in case "C" defaults. A typical stylized case occurs when "A" has bought a corporate bond issued from "C" and is waiting for the coupons and final notional payment from this bond: If "C" defaults before the corporate bond maturity, "A" does not receive such payments. "A" then goes to "B" and buys some protection against this risk, asking "B" a payment that roughly amounts to the bond notional in case "C" defaults.

Typically $\mathrm{L}_{\mathrm{GD}}$ is equal to a notional amount, or to a notional amount minus a recovery rate. We denote the recovery rate by "REC".

In exchange for this protection, company "A" agrees to pay periodically to "B" a fixed "running" amount $R$, at a set of times $\left\{T_{a+1}, \ldots, T_{b}\right\}, \alpha_{i}=T_{i}-T_{i-1}, T_{0}=0$. These payments constitute the "premium leg" of the CDS (as opposed to the LGD payment, which is termed the "protection leg"), and $R$ is fixed in advance at time 0; the premium payments go on up to default time $\tau$ if this occurs before maturity $T_{b}$, or until maturity $T_{b}$ if no default occurs.

$$
\begin{aligned}
& \text { "B" } \rightarrow \quad \text { protection } \mathrm{L}_{\mathrm{GD}} \text { at default } \tau_{C} \text { if } T_{a}<\tau_{C} \leq T_{b} \rightarrow \text { "A" } \\
& \text { "B" } \leftarrow \quad \text { rate } R \text { at } T_{a+1}, \ldots, T_{b} \text { or until default } \tau_{C} \leftarrow \text { "A" }
\end{aligned}
$$

Formally, we may write the RCDS ("R" stands for running) discounted value at 
time $t$ seen from "A" as

$$
\begin{aligned}
\prod_{\mathrm{RCDS}_{a}, b}(t):=-D(t, \tau)\left(\tau-T_{\beta(\tau)-1}\right) R 1_{\left\{T_{a}<\tau<T_{b}\right\}}- & \sum_{i=a+1}^{b} D\left(t, T_{i}\right) \alpha_{i} R 1_{\left\{\tau \geq T_{i}\right\}} \\
& +\mathbf{1}_{\left\{T_{a}<\tau \leq T_{b}\right\}} D(t, \tau) \mathrm{L}_{\mathrm{GD}}
\end{aligned}
$$

where $t \in\left[T_{\beta(t)-1}, T_{\beta(t)}\right)$, i.e. $T_{\beta(t)}$ is the first date among the $T_{i}$ 's that follows $t$, and where $\alpha_{i}$ is the year fraction between $T_{i-1}$ and $T_{i}$.

Sometimes a slightly different payoff is considered for RCDS contracts. Instead of considering the exact default time $\tau$, the protection payment $\mathrm{L}_{\mathrm{GD}}$ is postponed to the first time $T_{i}$ following default, i.e. to $T_{\beta(\tau)}$. If the grid is three or six months spaced, this postponement consists in a few months at worst. With this formulation, the CDS discounted payoff can be written as

$$
\prod_{\mathrm{PRCDS}_{a}, b}(t):=-\sum_{i=a+1}^{b} D\left(t, T_{i}\right) \alpha_{i} R \mathbf{1}_{\left\{\tau \geq T_{i}\right\}}+\sum_{i=a+1}^{b} \mathbf{1}_{\left\{T_{i-1}<\tau \leq T_{i}\right\}} D\left(t, T_{i}\right) \mathrm{L}_{\mathrm{GD}}
$$

which we term "Postponed Running CDS" (PRCDS) discounted payoff. Compare with the earlier discounted payout (10) where the protection payment occurs exactly at $\tau$ : The advantage of the postponed protection payment is that no accrued-interest term in $\left(\tau-T_{\beta(\tau)-1}\right)$ is necessary, and also that all payments occur at the canonical grid of the $T_{i}$ 's. The postponed payout is better for deriving market models of CDS rates dynamics and for relating CDS's to floaters, see for example Brigo (2004, 2004b). When we write simply "CDS" we refer to the RCDS case.

Let us consider again the basic RCDS: The pricing formula for this payoff depends on the assumptions on the interest rates dynamics and on the default time $\tau$. Let $\mathcal{F}_{t}$ denote the basic filtration without default, typically representing the information flow of interest rates and possibly other default-free market quantities (and also intensities in the case of reduced form models), and $\mathcal{G}_{t}=\mathcal{F}_{t} \vee \sigma(\{\tau<u\}, u \leq t)$ the extended filtration including explicit default information. In our current "structural model" framework with deterministic default barrier the two sigma-algebras coincide by construction, i.e. $\mathcal{G}_{t}=\mathcal{F}_{t}$, because here the default is completely driven by default-free market information. This is not the case with intensity models, where the default is governed by an external random variable and $\mathcal{F}_{t}$ is strictly included in $\mathcal{G}_{t}$, i.e. $\mathcal{F}_{t} \subset \mathcal{G}_{t}$.

We denote by $\operatorname{CDS}\left(t,\left[T_{a+1}, \ldots, T_{b}\right], T_{a}, T_{b}, R, \mathrm{~L}_{\mathrm{GD}}\right)$ the price at time $t$ of the above standard running CDS. At times some terms are omitted, such as for example the list of payment dates $\left[T_{a+1}, \ldots, T_{b}\right]$. In general we can compute the CDS price according to risk-neutral valuation (see for example Bielecki and Rutkowski (2001)):

$$
\operatorname{CDS}\left(t, T_{a}, T_{b}, R, \mathrm{~L}_{\mathrm{GD}}\right)=\mathbb{E}\left\{\prod_{\mathrm{RCDS}_{a}, b}(t) \mid \mathcal{G}_{t}\right\}=\mathbb{E}\left\{\prod_{\mathrm{RCDS}_{a}, b}(t) \mid \mathcal{F}_{t}\right\}=: \mathbb{E}_{t}\left\{\prod_{\mathrm{RCDS}_{a}, b}(t)\right\}
$$

in our structural model setup. A CDS is quoted through its "fair" $R$, in that the rate $R$ that is quoted by the market at time $t$ satisfies $\operatorname{CDS}\left(t, T_{a}, T_{b}, R, \mathrm{LGD}_{\mathrm{GD}}\right)=0$. Let us assume, for simplicity, deterministic interest rates; then we have

$$
\begin{array}{r}
C D S\left(t, T_{a}, T_{b}, R, \mathrm{~L}_{\mathrm{GD}}\right):=-R \mathbb{E}_{t}\left\{P(t, \tau)\left(\tau-T_{\beta(\tau)-1}\right) \mathbf{1}_{\left\{T_{a}<\tau<T_{b}\right\}}\right\} \\
-\sum_{i=a+1}^{b} P\left(t, T_{i}\right) \alpha_{i} R \mathbb{E}_{t}\left\{\mathbf{1}_{\left\{\tau \geq T_{i}\right\}}\right\}+\operatorname{LGD}_{\mathrm{GD}} \mathbb{E}_{t}\left\{\mathbf{1}_{\left\{T_{a}<\tau \leq T_{b}\right\}} P(t, \tau)\right\} .
\end{array}
$$


It is clear that the fair rate $R$ strongly depends on the default probabilities. The idea is to use quoted values of these fair $R$ 's with different maturities to derive the default probabilities assessed by the market.

While in simple intensity models the survival probabilities can be interpreted as discount factors (with credit spreads as discounting rates), and as such can be easily stripped from CDS's or corporate bonds, in structural models the situation is much more complicated. In fact, here, it is not possible to find a simple "credit spread" formulation for $d \mathbb{Q}\{\tau>t\}$ starting from (9). Moreover we have to pay attention to one fundamental aspect of the model. The barrier triggering default depends on the maturity chosen for a particular instrument, i.e. $H^{*}(t)=H_{T}^{*}(t)$ has a parametric dependence on $T$. But then, if we plan to use the model for instruments with different maturities, it is necessary to impose a consistency condition, that is

$$
H_{T_{1}}^{*}(t)=H_{T_{2}}^{*}(t)
$$

for $t \leq T_{1}<T_{2}$ for every pair of maturities $T_{1}$ and $T_{2}$. With our choice of $H^{*}(t)$ above we have easily that this condition implies, when enforced for all possible $T_{1}, T_{2}$ :

$$
r_{t}-q_{t}^{*}-(1+2 \beta) \frac{\sigma_{t}^{2}}{2}=0, \text { or } q_{t}^{*}=r_{t}-(1+2 \beta) \frac{\sigma_{t}^{2}}{2} .
$$

We notice that if this condition is satisfied, we have that the barrier $H^{*}$, defined in terms of $q^{*}$, obviously flattens to the constant value $H$. Thus consistency of the curved barrier for all maturities induces a flat barrier. In general, however, we expect (15) not to hold if $V^{*}$ is the value of the firm, since $r, q^{*}$ and $\sigma$ are given to us exogenously and $\beta$ is just constant. However, we can still manage to preserve analytical tractability as follows. Let us assume from now on that the real risk neutral dynamics of the firm value is a process given by

$$
d V_{t}=V_{t}\left(r_{t}-q_{t}\right) d t+V_{t} \sigma_{t} d W(t)
$$

where $q_{t}$ is the true payout ratio and $q_{t}^{*}$ is defined by (15). The "system" $\left(V^{*}, H^{*}\right)$ with said $q^{*}$ is both tractable and satisfying the consistency condition. Our problem is that $V^{*}$ is not the real firm value, so that the system $\left(V^{*}, H^{*}\right)$ is not good for modeling default. However, if we define

$$
\widehat{H}(t)=H \exp \left(-\int_{0}^{t}\left(q_{s}-q_{s}^{*}\right) d s\right)
$$

by integrating $V^{*}$ and $V^{\prime}$ 's equations it is easy to show that the first time $V_{t}^{*}$ hits $H^{*}(t)=H$ is the same as the first time the real process $V_{t}$ hits $\widehat{H}(t)$. Therefore, default probabilities computed with the tractable model $V^{*}, H^{*}$ are the same as default probabilities for the "true" model $V, \widehat{H}$. We can compute quantities and perform our calibration with the former model and consider the latter as the real model. We can thus state the following

Proposition 3.1. (Analytically-Tractable First Passage (AT1P) Model) Assume the risk neutral dynamics for the value of the firm $V$ is characterized by a risk free rate $r_{t}$, a payout ratio $q_{t}$ and an instantaneous volatility $\sigma_{t}$, according to equation (16), i.e.

$$
d V_{t}=V_{t}\left(r_{t}-q_{t}\right) d t+V_{t} \sigma_{t} d W(t)
$$


and assume a default barrier $\widehat{H}(t)$ of the form given in (17) with $q^{*}$ given as in (15), i.e.

$$
\widehat{H}(t)=H \exp \left(-\int_{0}^{t}\left(q_{s}-r_{s}+(1+2 \beta) \frac{\sigma_{s}^{2}}{2}\right) d s\right)
$$

and let $\tau$ be defined as the first time where $V$ hits $\widehat{H}$ from above, starting from $V_{0}>H$,

$$
\tau=\inf \left\{t \geq 0: V_{t} \leq \widehat{H}(t)\right\}
$$

Then the survival probability is given analytically by

$$
\mathbb{Q}\{\tau>T\}=\left[\Phi\left(\frac{\log \frac{V_{0}}{H}+\beta \int_{0}^{T} \sigma_{s}^{2} d s}{\sqrt{\int_{0}^{T} \sigma_{s}^{2} d s}}\right)-\left(\frac{H}{V_{0}}\right)^{2 \beta} \Phi\left(\frac{\log \frac{H}{V_{0}}+\beta \int_{0}^{T} \sigma_{s}^{2} d s}{\sqrt{\int_{0}^{T} \sigma_{s}^{2} d s}}\right)\right] .
$$

Formula (18) is easily obtained by substituting the consistency condition (15) in formula (9) for the survival probability. From our earlier definitions, straightforward computations lead to the price at initial time 0 of a CDS, under deterministic interest rates, as

$$
\begin{gathered}
\operatorname{CDS}_{a, b}\left(0, R, \mathrm{~L}_{\mathrm{GD}}\right)=R \int_{T_{a}}^{T_{b}} P(0, t)\left(t-T_{\beta(t)-1}\right) d \mathbb{Q}(\tau>t) \\
-R \sum_{i=a+1}^{b} P\left(0, T_{i}\right) \alpha_{i} \mathbb{Q}\left(\tau \geq T_{i}\right)-\mathrm{L}_{\mathrm{GD}} \int_{T_{a}}^{T_{b}} P(0, t) d \mathbb{Q}(\tau>t)
\end{gathered}
$$

so that if one has a formula for the curve of survival probabilities $t \mapsto \mathbb{Q}(\tau>t)$, as in our AT1P structural model, one also has a formula for CDS.

Notice an important feature of Formula (18) and then (19): Survival (and default) probabilities and CDS values only depend on the ratio between $V$ and $H$, and not on $V$ and $H$ separately. This means that, as far as default probabilities are concerned, a precise estimation of $V$ is not needed. We can express default probabilities in relative terms, i.e. in terms of $V / H$. In other terms, we can imagine to re-scale $V$ (considering $V / V_{0}$ so that the initial condition reads $V_{0} / V_{0}=1$ ) and also the barrier parameter $H$ (taking $H / V_{0}$, a number smaller than one as barrier).

Formula (18) can be used to fit the model parameters to market data. However, the only parameter left that can account for time dependence is the volatility. If we use exogenous volatility (deduced perhaps from historical or implied equity volatility) we are left with no freedom. However, we may infer the first year volatility $\sigma(0 \div 1 y):=$ $\{\sigma(t): t \in[0,1]\}$ from equity data and use $H$ as a first fitting parameter, and then use the remaining later volatilities $\sigma(1 y \div 2 y), \sigma(2 y \div 3 y)$ etc as further fitting parameters. Therefore $\sigma(2 y \div \cdot)$ will be determined by credit quality as implied by CDS data rather than by equity data. To sum up, we can choose piecewise constant volatility, and look for those volatility values after the first year that make the quoted CDS's fair when inserting in their premium legs the market quoted $R$ 's. In this way we find as many volatilities as many CDS's we consider minus one. In this first case $H$ is determined by CDS's and we explain below how exactly we find this $H$ (credit spread method).

Alternatively, if we aim at creating a one to one correspondence to volatility parameters and CDS quotes, we can exogenously choose the value $H$ (for example we 
will see below the protection/excursion method, but judgemental analysis or trial and error are often needed) and $\beta$, leaving all the unknown information in the calibration of the volatility. If we do so, we find exactly one volatility parameter for each CDS maturity, including the first one.

In general the above CDS calibration procedures are justified by the fact that in the end we are not interested in estimating the real process of the firm value underlying the contract, but only in reproducing risk neutral default probabilities with a model that makes sense also economically. While it is important that the underlying processes have an economic interpretation, we are not interested in sharply estimating them or the capital structure of the firm, but rather we appreciate the structural model interpretation as a tool for assessing the realism of the outputs of calibrations, and as an instrument to check economic consequences and possible diagnostics.

Finally, in case we still have a preferred terminal maturity $\bar{T}$ for the debt, and an indication of the final debt at maturity, which we call $L$, it makes sense to impose that our barrier $\widehat{H}$ be always below the present value of the final debt, i.e. $P(t, \bar{T}) L$. This condition amounts to assuming that we are cutting some slack to the firm by allowing it to go somehow below the debt present value before forcing it to declare bankruptcy. How much below can be decided by means of $H$ and $\beta$. In detail, our condition reads

$$
H \exp \left(-\int_{0}^{t}\left(q_{s}-r_{s}+(1+2 \beta) \frac{\sigma_{s}^{2}}{2}\right) d s\right)<\exp \left(-\int_{t}^{\bar{T}} r_{s} d s\right) L
$$

We can easily rewrite this condition as

$$
L>H \exp \left[-\int_{0}^{t}\left(q_{s}+(1+2 \beta) \frac{\sigma_{s}^{2}}{2}\right) d s+\int_{0}^{\bar{T}} r_{s} d s\right]
$$

A sufficient condition for this, in case the round brackets term is positive, is

$$
H \leq L P(0, \bar{T})
$$

i.e. the final debt initial present value has to larger than the barrier parameter $H$. This means that in all our use of the model with positive round brackets one may presume a final debt $L$ at a preferred maturity (typically larger than ten years, since this is the largest CDS maturity) satisfying this condition.

We are still in need to connect equity and firm value if we aim at deriving part of the value of the firm volatility from equity data. Since default is enforced as soon as $V$ hits $\widehat{H}$ at time $\tau$ if before $\bar{T}$, or is given at $\bar{T}$ if $V_{\bar{T}}$ is below $L$, the debt value at time $t$ would be

$$
D_{t}=\mathbb{E}_{t}\left[D(t, \tau) \widehat{H}(\tau) \mathbf{1}_{\{\tau<\bar{T}\}}\right]+\mathbb{E}_{t}\left[D(t, \bar{T}) \min \left(L, V_{\bar{T}}\right) \mathbf{1}_{\{\tau \geq \bar{T}\}}\right]
$$

which can be computed as a function of $V_{t}$ with computations similar to (18) (with time $t$ replacing time 0). In turn, we would have the equity value as $S_{t}=V_{t}-D_{t}=$ $V_{t}-D_{t}\left(V_{t}\right)$ from which we could derive an approximation for $V$ 's (unknown) volatility in terms of $S$ 's (known, be it historical or implied) volatility through Ito's formula and some approximations. 
However, we will not pursue this strategy but simply take the equity volatility itself as a proxy for the order of magnitude of the firm value volatility. As a matter of fact, our preferred approach will be to let CDS data select most (and in some cases all) values of the firm volatilities.

\subsection{First Numerical Example}

In this section we present some results of the calibration performed with the structural model. We consider CDS contracts having the telecoms sector Vodafone company as underlying with recovery rate $\mathrm{R}_{\mathrm{EC}}=40 \%\left(\mathrm{~L}_{\mathrm{GD}}=0.6\right)$. In Table 1 we report the maturities $T_{b}$ of the contracts and the corresponding "mid" CDS rates $R_{0, b}^{\mathrm{MID}}(0)$ (quarterly paid) on the date of March 10th, 2004, in basis points $\left(1 b p=10^{-4}\right)$. We take $T_{a}=0$ in all cases.

\begin{tabular}{|c|c|}
\hline Maturity $T_{b}$ & Rate $R_{0, b}^{\mathrm{MID}}(0)(\mathrm{bps})$ \\
\hline March 21st, 2005 & 21.5 \\
March 20th, 2007 & 33.0 \\
March 20th, 2009 & 43.0 \\
March 21st, 2011 & 49.0 \\
March 20th, 2014 & 61.0 \\
\hline
\end{tabular}

Table 1: Maturities of Vodafone's CDS's with their corresponding rates on March 10, 2004.

In Tables 2 and 3 we present the results of the calibration performed with the structural model and, as a comparison, of the calibration performed with a deterministic intensity (credit spread) model (using piecewise linear intensity). In this first example the parameters used for the structural model have been selected based on qualitative considerations, and are $\beta=0.5$ and $H / V_{0}=0.5$. The $\sigma$ 's have been found by calibration to CDS quotes. Below we report both the values of the calibrated parameters in the two models (volatilities and intensities) and the related survival probabilities.

\begin{tabular}{|c|c|c|}
\hline Maturity $T_{b}$ & Volatility $\sigma\left(T_{b-1} \div T_{b}\right)$ & Survival Prob. $\mathbb{Q}\left(\tau>T_{b}\right)$ \\
\hline March 10th, 2004 & $24.343 \%$ & $100.000 \%$ \\
March 21st, 2005 & $24.343 \%$ & $99.625 \%$ \\
March 20th, 2007 & $12.664 \%$ & $98.315 \%$ \\
March 20th, 2009 & $12.766 \%$ & $96.352 \%$ \\
March 21st, 2011 & $12.659 \%$ & $94.204 \%$ \\
March 20th, 2014 & $15.271 \%$ & $89.645 \%$ \\
\hline
\end{tabular}

Table 2: Calibrated piecewise constant volatilities nodes and subsequent survival probabilities with the structural model. In the first column there are the maturities of the contracts (the first entry is the date where the calibration is performed. All $\sigma$ 's have been obtained from CDS quotes, $H$ and $\beta$ being fixed exogenously).

As a further comparison, we plot the behavior of the survival probabilities $T \mapsto$ $\mathbb{Q}\{\tau>T\}$ resulting from CDS calibration in both cases (Figure11). It is clear that the 


\begin{tabular}{|c|c|c|}
\hline Maturity & Intensity & Survival Prob. $\mathbb{Q}\left(\tau>T_{b}\right)$ \\
\hline March 10th, 2004 & $0.357 \%$ & $100.000 \%$ \\
March 21st, 2005 & $0.357 \%$ & $99.627 \%$ \\
March 20th, 2007 & $0.952 \%$ & $98.316 \%$ \\
March 20th, 2009 & $1.033 \%$ & $96.355 \%$ \\
March 21st, 2011 & $1.189 \%$ & $94.206 \%$ \\
March 20th, 2014 & $2.104 \%$ & $89.604 \%$ \\
\hline
\end{tabular}

Table 3: Intensity model. In the second column we present the nodes of the piecewise linear calibrated intensities and in the last column the corresponding survival probabilities.

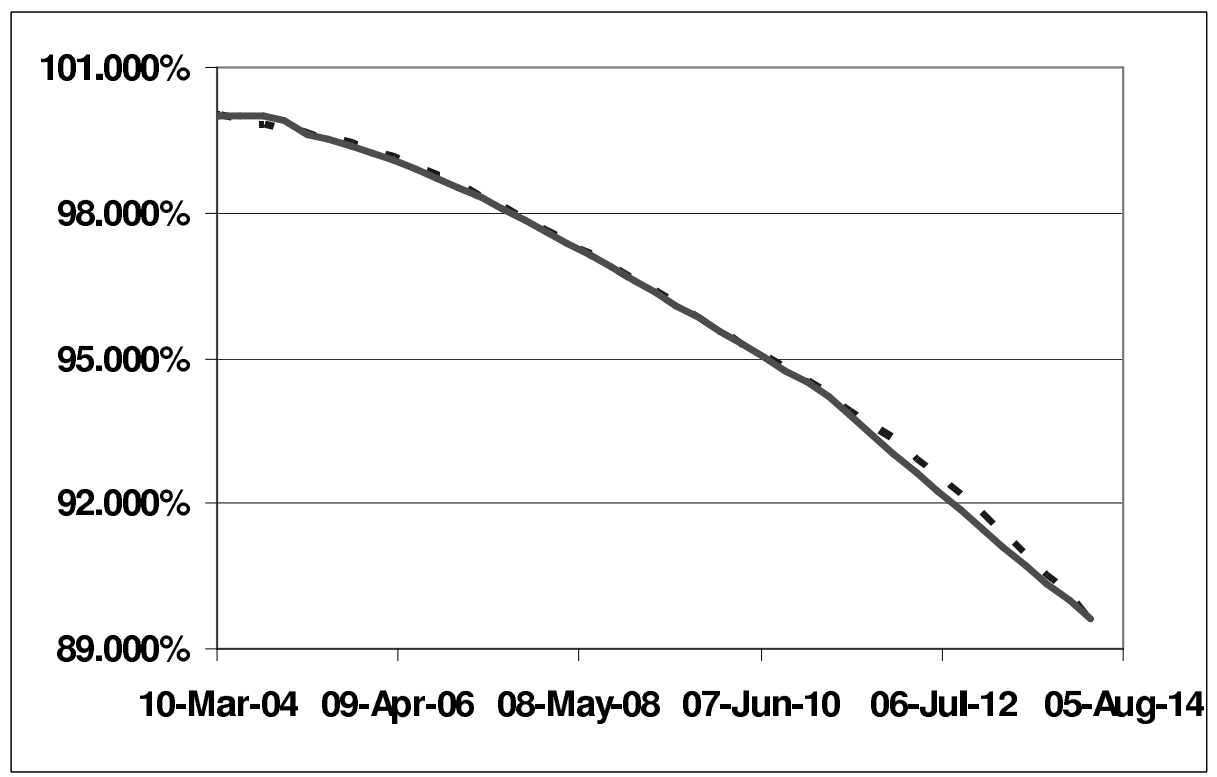

Figure 1: Survival probabilities $T \mapsto \mathbb{Q}\{\tau>T\}$ comparison between the CDS calibration with the structural model and the CDS calibration with the intensity model. The dotted line corresponds to survival probabilities computed with the intensity model, the continuous line to survival probabilities computed with the structural model.

survival probability found with the two different models is nearly the same, which indirectly says that CDS are actually instruments that efficiently translate default probabilities into prices, since the default probabilities depend little on the chosen model, as long as this is consistent with the same CDS quotes. This is somehow obvious when keeping in mind (19).

A difference is in the first period, i.e. during the life of the first CDS, where the probability computed with the structural model is slightly above the other one, given the less sudden nature of the default in structural models in general. This is illustrated in Figure 1. This leads us to the following

Remark 3.2. (Short term credit spreads). It is often said that structural models imply unrealistic short-term credit spreads. However, if "short-term" is meant as a realistic short maturity, our model does not suffer from this drawback, since it can calibrate any realistic $6 m$ or hypothetical $3 \mathrm{~m} C D S$ quote. If one is not happy with the default probability between 0 and $1 y$, it suffices to calibrate a shorter term $C D S$, 
and the model can do this exactly. What is more, we will see below a case with very high default probability where only the structural model is able to calibrate the CDS quotes, the intensity model giving negative intensities as outputs.

In Figure 2 we plot the term structure of the calibrated volatility (see also Table2). Sometimes the calibration yields values that are not plausible as volatility levels, but these volatilities are to be taken as default probabilities fitting parameters with an economic interpretation rather than directly as firm value volatilities. In any case, consistency with CDS quotes for relative value pricing is an important aspect we need to incorporate in any relative value pricing model. Also, embedding CDS information in a structural model framework allows us to check a posteriori the realism of the calibrated quotes and of the model, so that we may play with our degrees of freedom (for example in $\beta$ and $H$ ) to obtain more realistic calibrations.

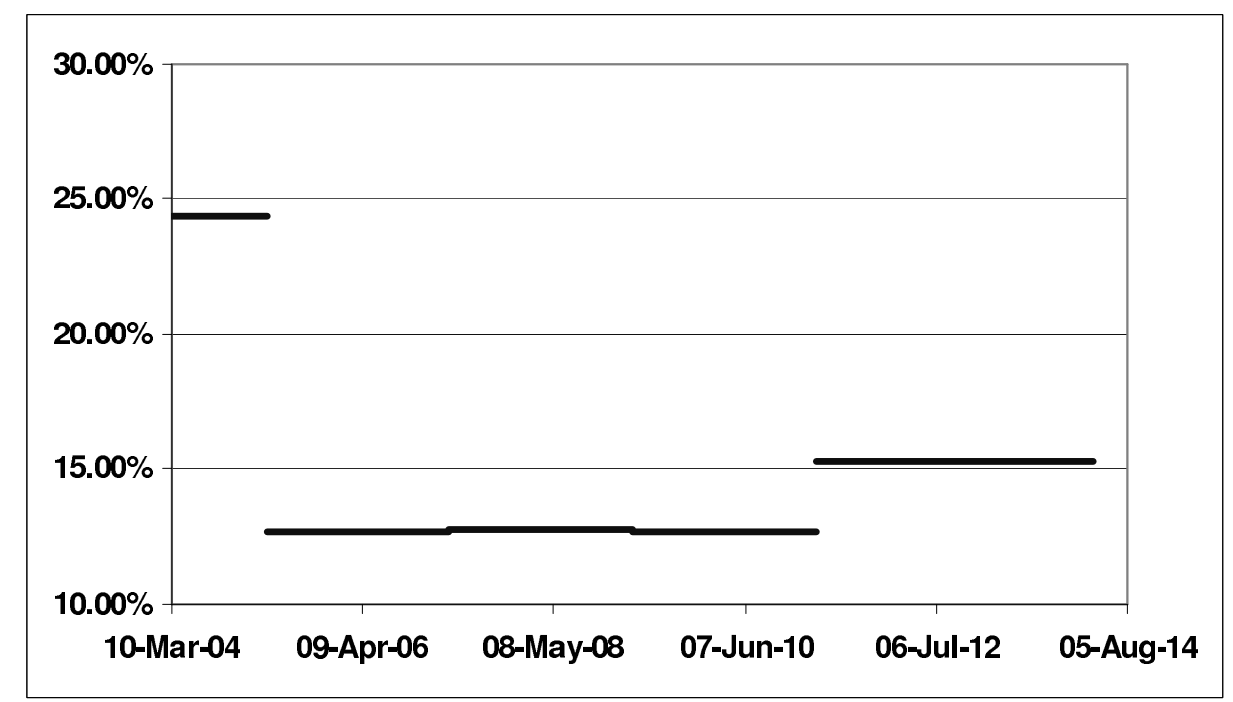

Figure 2: Volatility term structure $T \mapsto \sigma(T)$ obtained from the calibration. The corresponding data are presented in Table 2 ,

We can double check the validity of our results above by means of a Monte Carlo simulation. We can simulate numerically the process $V^{*}$ and check whether it crosses the default barrier $H^{*}$ or not: In both cases we can compute the (discounted) payoff of the CDS for that particular path. We repeat this procedure $N$ times and find the expected value of the payoff as a sample mean from the simulated scenarios. If, for each maturity $T_{b}$ we use the rate $R_{0, b}(0)$ given by the market, then we should obtain CDS's with zero values.

First of all we derive the right dynamics for the underlying: We use the process (1) using the consistency condition (15), obtaining

$$
d V_{t}^{*}=V_{t}^{*}(1+2 \beta) \frac{\sigma_{t}^{2}}{2} d t+V_{t}^{*} \sigma_{t} d W(t)
$$

We know that in general simulations of processes with barriers require a very large number of paths and a very small time step. To avoid this problem we applied the Brownian Bridge method (see for example Metwally and Atiya (2002)), which allows 
us to use a larger time step (actually we used an interval of about five days), thus reducing significantly the simulation time without losing precision on the reproduced default probabilities.

In Table 4 we present the result of the Monte Carlo simulation. We used the same data considered at the beginning of the section, and the rates $R$ given in Table 1 . We used $N=250000$ scenarios, finding that the values of the CDS's simulated according to the analytically calibrated dynamics are practically zero as should be (zero is inside the small standard error MC window).

\begin{tabular}{|c|c|c|}
\hline Maturity & Simulated Payoff & MC std. dev. \\
\hline March 21st, 2005 & 0.2 & 0.7 \\
March 20th, 2007 & 0.7 & 1.5 \\
March 20th, 2009 & -0.9 & 2.1 \\
March 21st, 2011 & -0.8 & 2.5 \\
March 20th, 2014 & -0.1 & 3.1 \\
\hline
\end{tabular}

Table 4: Simulated payoffs of the previously considered CDS's in basis points. The errors of the Monte Carlo simulations are reported as well. The number of scenarios is $N=250000$.

\subsection{Finding $H$ : Credit spread method and protection/excursion analogy method}

Not all possible values of the parameters $H$ and $\beta$ are good for the calibration. The parameter $\beta$ can be used to shape the safety covenants barrier $\widehat{H}$ once $q, \sigma$ and $r$ are given. And if we calibrate the $\sigma$ 's, the initial choice of $\beta$ will have repercussions on the calibration output patterns. In general for a given $\beta$, if $H$ is too high (or too low) the calibration may not succeed in finding all the volatilities. A way to choose a valid reference value $H$ having also a more direct link with market information is the following. Suppose we know the equity volatility for one-year maturity (for example an implied volatility taken from quoted options) and suppose we set the structural model's $\sigma$ up to one year to a constant value given by said equity volatility. Then the probability of not defaulting before $T$ (=1 year) is given by (see (18))

$$
\mathbb{Q}\{\tau>T\}=\left[\Phi\left(\frac{\log \frac{1}{H}+\beta \sigma^{2} T}{\sigma \sqrt{T}}\right)-H^{2 \beta} \Phi\left(\frac{\log H+\beta \sigma^{2} T}{\sigma \sqrt{T}}\right)\right]=\psi(H ; \beta, T, \sigma)
$$

(we have re-scaled $V$ to one, i.e. $V_{0}=1$, so that the found $H$ will have to be multiplied by $V_{0}$ ) and depends on $H$. Being all other parameters known, $\psi$ is monotonically decreasing in $H$. But if we adopt a deterministic intensity model for one moment, the market provides us with

$$
\mathbb{Q}\{\tau>T\}=\exp \left(-\int_{0}^{T} \lambda_{C D S}(s) d s\right)
$$

where $\lambda_{C D S}$ are the deterministic intensities that have been stripped from CDS data. To impose a reasonable barrier $H$ we solve

$$
\exp \left(-\int_{0}^{T} \lambda_{C D S}(s) d s\right)=\psi(H ; \beta, T, \sigma)
$$


in $H$. Since $\psi$ is monotonic, this should be easily dealt with numerically. We may call this value the "credit spread based $H$ ", since $H$ comes from a default probability based on credit spreads stripped by CDS's. Using this $H$, we calibrate the structural model volatilities to the CDS quotes, including the first one. Since the intensity-based 1y default probability extracted from CDS and the CDS quote itself contain similar information but are not exactly equivalent, we will find a $\sigma(0 \div 1 y)$ that is close to our initial one-year $\sigma$ selected from the equity market, but not exactly the same. This is due for example to the first CDS having quarterly payments on whose dates survival probabilities depend on the chosen model. Even if two models agree on the one-year survival probability, they are not necessarily agreeing on the say semiannual default probability. And if a payoff involves said default probability the two models may give slightly different values even if they agree on the one-year default probability. This is why for example in Table 7 we find $\sigma(0 \div 1 y)=5.012 \%$ when the equity volatility is $5 \%$.

A different possibility for choosing $H$ is the following. We may compare the range of protection $\mathrm{L}_{\mathrm{GD}}$ offered with the CDS to a rough covering of the excursion $V-H$ characterizing default in the structural model. If we consider then $\mathrm{LGD}_{0} V_{0}$ as the protection (on the notional $V_{0}$ associated with the initial value of the firm) and set it equal to $V_{0}-H$ we find

$$
\mathrm{L}_{\mathrm{GD}}=1-H / V_{0}, \quad \text { or } H / V_{0}=\mathrm{R}_{\mathrm{EC}},
$$

so that we have an immediate candidate for $H$. We term this value the "excursion/protection analogy $H$ ". However, if we resort to this value we may have numerical problems in stripping volatilities and moreover we cannot impose the one-year volatility from the equity market any longer, since we have already used the degree of freedom provided by $H$. Indeed the calibration proceeds in the same way as before, but problems may arise in low recovery cases. Low values for recovery rates imply low barriers, which in turn lead to high values for stripped volatilities, even much larger than $100 \%$. Under these conditions there are times when the solver is not able to retrieve a feasible solution for the calibrated volatilities. A way to bypass this obstacle is given by the parameter $\beta$ : its main role is to vary the steepness of the safety covenant barrier, as is clear from (17), and this fact has a relevant impact also on the term structure of volatilities resulting from the calibration, as we explain more in detail in the following section. As a matter of fact, it is possible to indirectly act on the volatilities by varying $\beta$, looking for those values of $\beta$ returning reasonable values of $\sigma$ from the calibration, while keeping an eye on the realism of the resulting safety barrier.

\section{Further Model Specification and a Case Study: the Parmalat Crisis}

We now apply the model to a concrete case. We are going to see what happens, in terms of model parameters, when a firm approaches default. In particular we consider the case of Parmalat Finanziaria SpA, an important Italian company whose main activity is the production of milk and other alimentary goods, and also present in a large variety of other sectors. In the past years this company and the related 
stock have been very important for the Italian economy; in fact in 2003 the Parmalat stock was included in the main Italian stock index (Mib 30). After some signal worrying the investors, at the end of 2003 the Italian control commission (Consob) began to analyze deeply the balance sheets of the company, and as the investigation proceeded the company entered a deep crisis.

Here we plan to analyze how the structural model behaves in such a situation, as the credit quality implied by CDS quotes deteriorates in time. First of all, in Figure 3 we present the graph of the stock price and the graph of the (1y-) CDS rate $R$. It is immediate to see that there exists a sort of negative correlation between the two, as expected: As the equity value $S$ increases, the CDS premium $R$ (expressing a protection cost) decreases. From the historical data, we see that the stock price experienced a deep fall on December 11th. So we consider some particular dates before that day, some in "standard regime conditions" and also some dates in "crisis conditions", to see how the CDS calibration changes with market conditions.

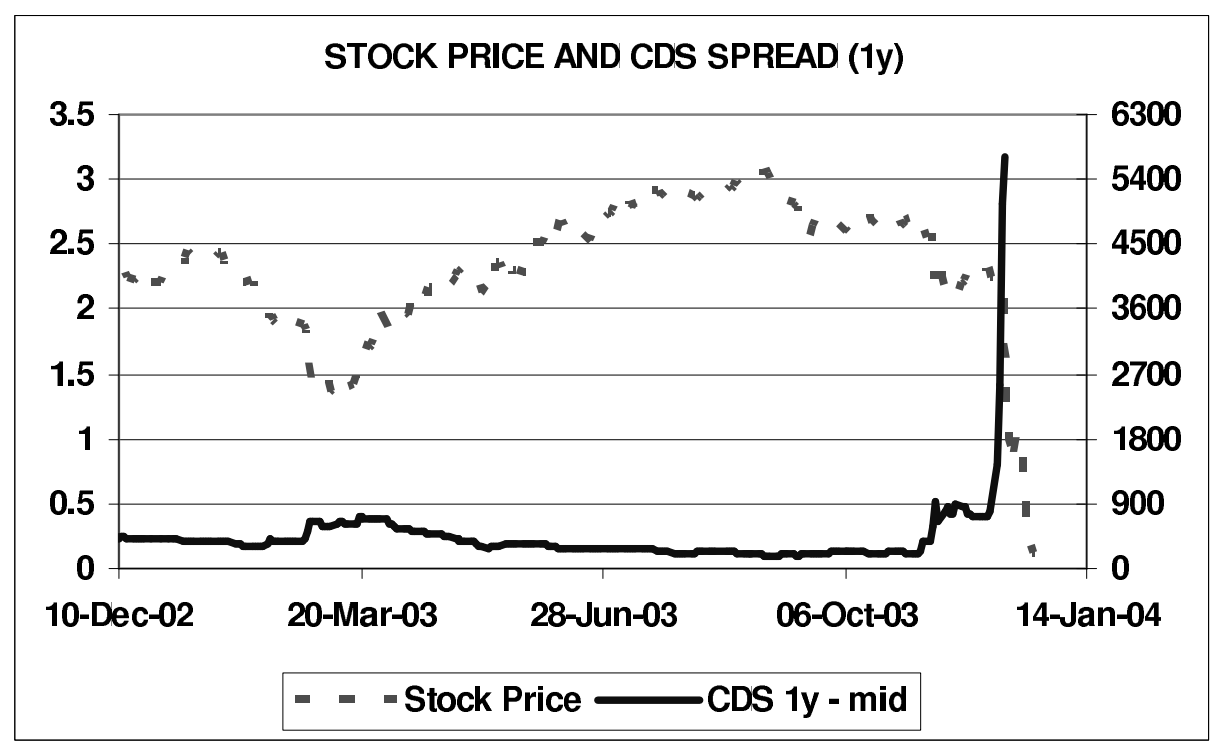

Figure 3: Historical behavior of the Parmalat stock $S_{t}$ (in euros) and of the 1y-CDS $R(t)$ (in bps). The dotted line corresponds to the stock price (left scale) while the continuous line to the CDS rate (right scale).

In Table 5 we report the considered dates, with the related stock prices, CDS rates (in basis points), and the recovery rates. We also add the volatilities: These are historical volatilities obtained by the last month of data (as records of implied volatility were not available). These volatilities have been sometimes approximated in excess, to take into account the fact that in crisis periods the stock becomes more volatile than in the previous period, an effect that is not modelled by historical volatility.

Some important remarks on the data. First of all we see that the CDS rates $R$ (which were already high in the first dates we considered) grow very fast and the recovery rate decreases as the crisis approaches. The historical volatility we use as a proxy for $1 \mathrm{y}$ volatility increases a lot in time as well (probably the last value of $50 \%$ is even underestimated, considering that after a couple of days the price has gone below one euro). We have also set the payout ratio identically equal to zero. 


\begin{tabular}{|c|c|c|c|c|}
\hline Date & Sep 10th & Nov 28th & Dec 8th & Dec 10th \\
\hline R (1y) & 192.5 & 725 & 1450 & 5050 \\
R (3y) & 215 & 630 & 1200 & 2100 \\
R (5y) & 225 & 570 & 940 & 1500 \\
R (7y) & 235 & 570 & 850 & 1250 \\
R (10y) & 235 & 570 & 850 & 1100 \\
REC & 40 & 40 & 25 & 15 \\
S & 2.898 & 2.297 & 2.237 & 2.237 \\
$\sigma(\%)$ & 5 & 14 & 20 & 50 \\
\hline
\end{tabular}

Table 5: Input data.

With this data, we are now able to compute the credit spread based barrier parameter $H$ for each considered date. In principle we could plot the value $H$ on a graph with the corresponding initial value $V_{0}$ (either normalized or not), and also the behavior of the real barrier $\widehat{H}$. Even if we did this for each date, this would not be sufficient to deduce whether the model efficiently incorporates default information because the different volatilities may be misleading. More precisely, for normalized values about 1 we find $H=0.8977$ for September 10th and $H=0.7253$ for December 10th. As the first safety barrier is closer to the initial value $V_{0}=1$, one could think that the default is more likely in the first situation, but we know that this is not the case. The fact is that in the first situation we have a much lower volatility than in the second. So we decided to plot not only the barrier $\widehat{H}$ and the initial value of the firm $V_{0}$, but also the expected price in the future $t \mapsto \mathbb{E}\left(V_{t}\right)$ and a confidence region $t \mapsto \exp \left(\mathbb{E}\left[\ln \left(V_{t}\right)\right]-\operatorname{Std}\left[\ln \left(V_{t}\right)\right]\right)$ and $t \mapsto \exp \left(\mathbb{E}\left[\ln \left(V_{t}\right)\right]+\operatorname{Std}\left[\ln \left(V_{t}\right)\right]\right)$, where Std is the risk neutral standard deviation. Std is easily computed for normal variates such as $\ln (V)$. All these graphs are reported in Figures from 4 to 7 . In the confidence region between the expected price minus/plus one standard deviation there are the $68 \%$ of the total scenarios. Hence the instant in which the lower bound of the confidence region hits the barrier is a signal of the proximity of the default. And from the graphs it is clear that as time passes, the default becomes more and more likely and the scenarios trajectories widen, hitting the barrier earlier. Indeed, if on September 10th the confidence region hits the barrier after nearly ten years, on December 10th this happens after less than one year. At this point of the discussion a few remarks on the choice of $\beta$ are in order.

Putting the consistency condition (15) in the equation for the barrier $\widehat{H}$ given in (17), after some calculations we find

$$
\widehat{H}(t)=\frac{H}{V_{0}} \mathbb{E}\left[V_{t}\right] \exp \left[-\frac{(1+2 \beta)}{2} \int_{0}^{t} \sigma_{s}^{2} d s\right] .
$$

Remark 4.1. The role of the $\beta$ parameter. The integral of the squared volatility has the effect of introducing a concave and decreasing factor into the shape of the safety barrier, provided the round brackets term is positive. The role of $\beta$ is thus clear: For given values of the volatilities $\sigma, \beta$ is the parameter that when increased allows us to increase the concavity and the steepness of the decreasing factor in the barrier, thus lowering it. This parameter may be useful when the calibrated volatilities get out 
of control. Indeed, in case the needed concavity is high and $\beta$ is small, volatilities may tend to explode to obtain the required barrier factor. By increasing $\beta$ the volatilities needed to produce a given steepness and concavity can become smaller, avoiding this risk.

In our first experiments we choose $\beta=0.5$ such that the argument of the exponential is simply the integral of the variance, but this is just our particular choice based on numerical experiments. In this case, for low values of the volatilities the barrier is very similar to the behavior of the expected price, hence it is upward sloped. On the contrary, high values of the volatilities change significantly the shape of the barrier, but also the confidence region widens as a consequence. These effects can be checked by comparing Figures from 4 to 7 to the Tables reported in the Appendix. In Figure 8 we show a typical term structure of volatility obtained from the calibration, corresponding to the data of December 8th.

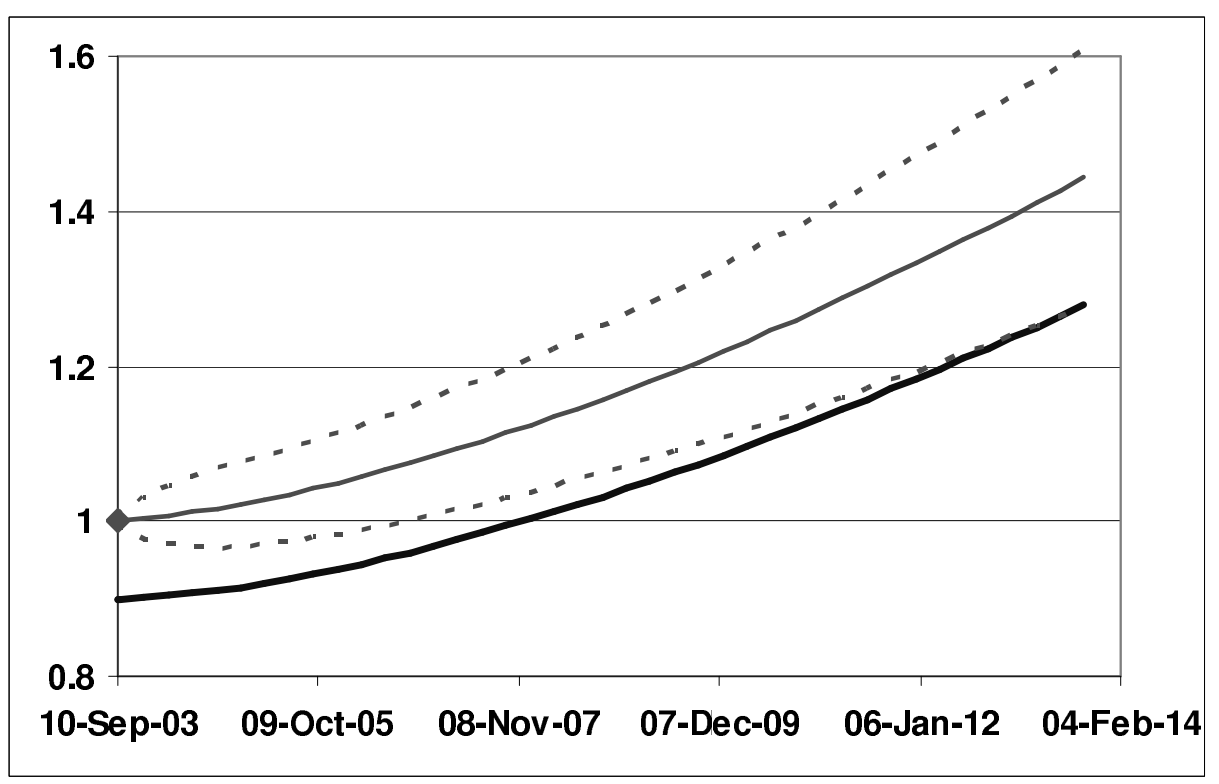

Figure 4: Default barrier $t \mapsto \widehat{H}(t)$ on September 10th, 2003, $t \mapsto \mathbb{E}\left(V_{t}\right)$ and $t \mapsto$ $\exp \left(\mathbb{E}\left[\ln \left(V_{t}\right)\right] \mp \operatorname{Std}\left[\ln \left(V_{t}\right)\right]\right)$.

Furthermore, in Figure 9 we plot the survival probability on December 10th. We see that the probability falls down very quickly in the first period, but later it decreases in a much slower way. This fact can be explained as follows. When the firm is approaching default, investors are more interested in buying protection over a small time horizon, because they assess a very high probability to the default event for the immediate future period. But if the firm survives to the full crisis, probably it will reach a certain stability situation, characterized by a "lower" default probability, so that provided the company survives the first immediate times after the crisis, investors are less worried by subsequent periods.

As a final investigation, we have repeated the calibration when $H$ is selected under the alternative method of the "excursion/protection analogy", i.e. by setting $H / V_{0}=$ $R_{\text {ec }}$. In the Appendix we report all the results. The first important remark is that here we have used $\beta=0.08$. This is due to the fact that the calibration for the last date 


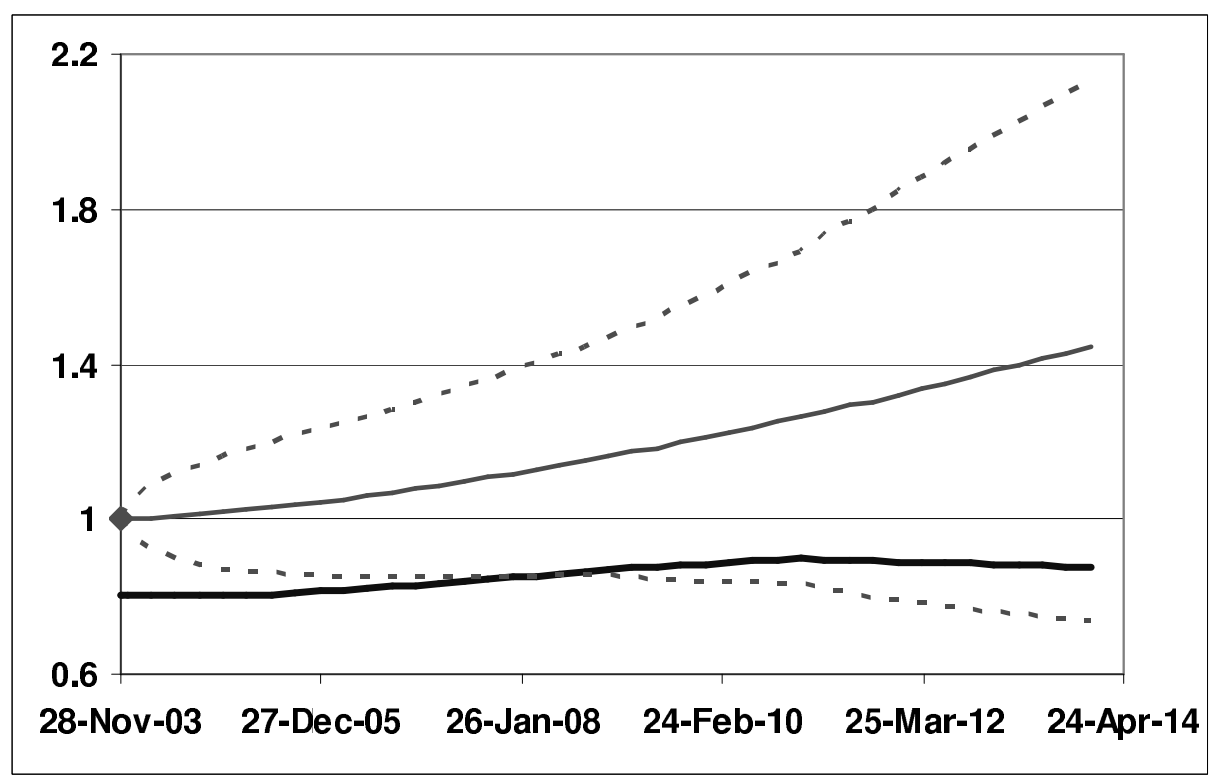

Figure 5: Same as above on November 28th, 2003.

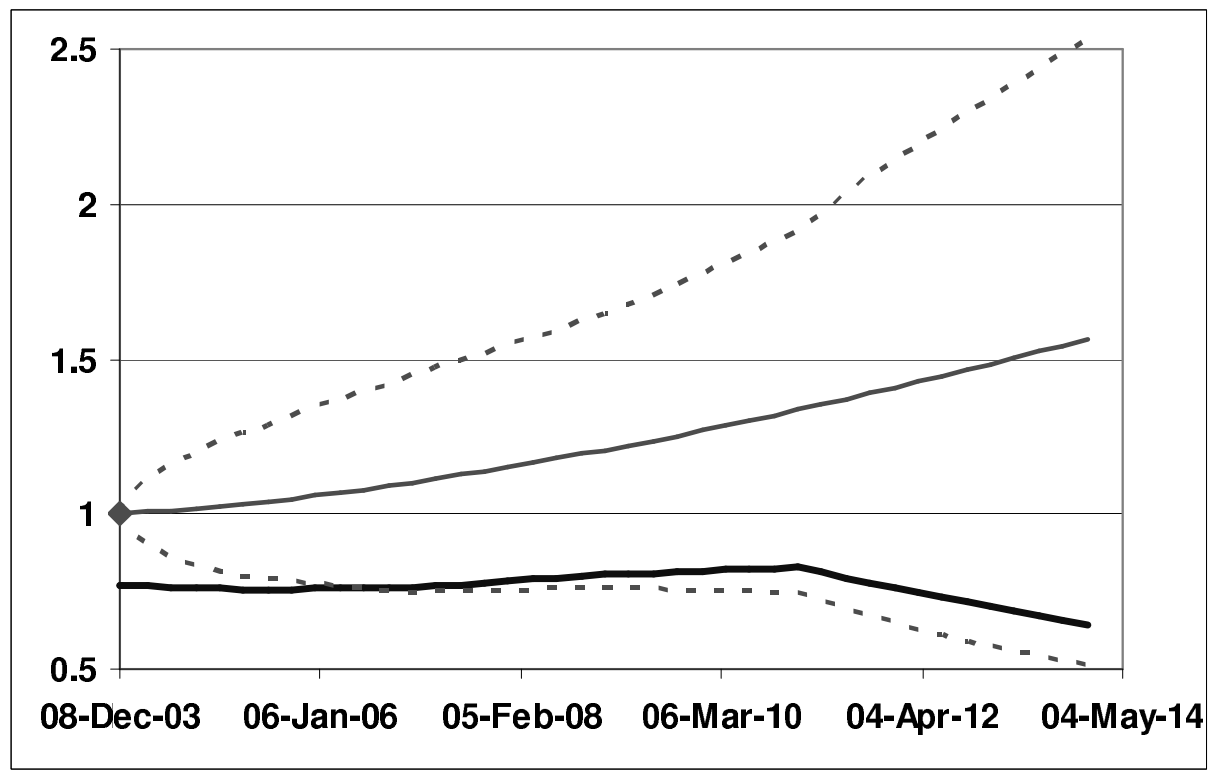

Figure 6: Same as above on December 8th, 2003.

requires very high volatilities, being the relevant $H$ very low. Then we are compelled to use low $\beta$ 's to avoid the calibration explosion hinted at in Remark 4.1. At the same time we cannot diminish $\beta$ too much, because the second volatility $\sigma(2 y \div 3 y)$ is very low due to the peculiar structure of CDS market quotes across maturity. Ceteris paribus, diminishing $\beta$ means raising the barrier and thus diminishing volatilities, but $\sigma$ has a lower bound equal to zero. So, in the end, the feasible interval for the $\beta$ values is rather narrow and $\beta=0.08$ is one value in this narrow interval. For previous dates, the calibration was possible also with other values of $\beta$ but we decided to keep it fixed to 0.08 for uniformity reasons. 


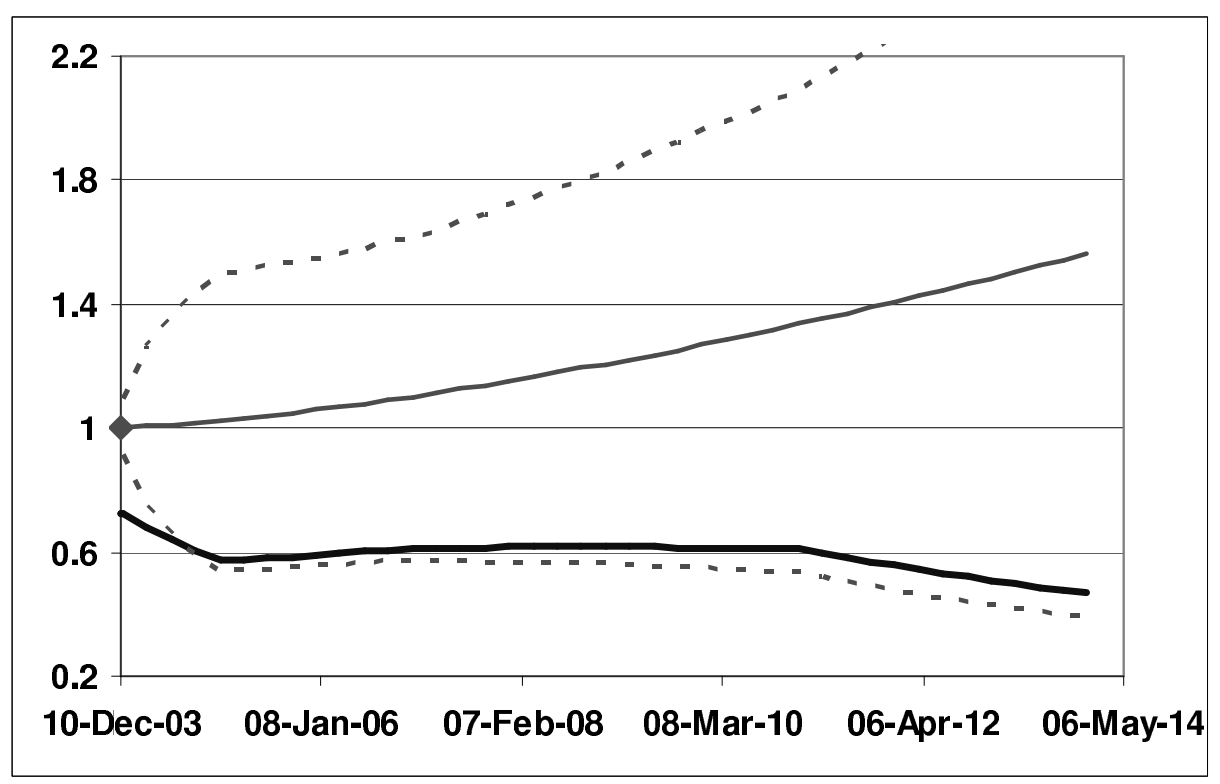

Figure 7: Same as above on December 10th, 2003.

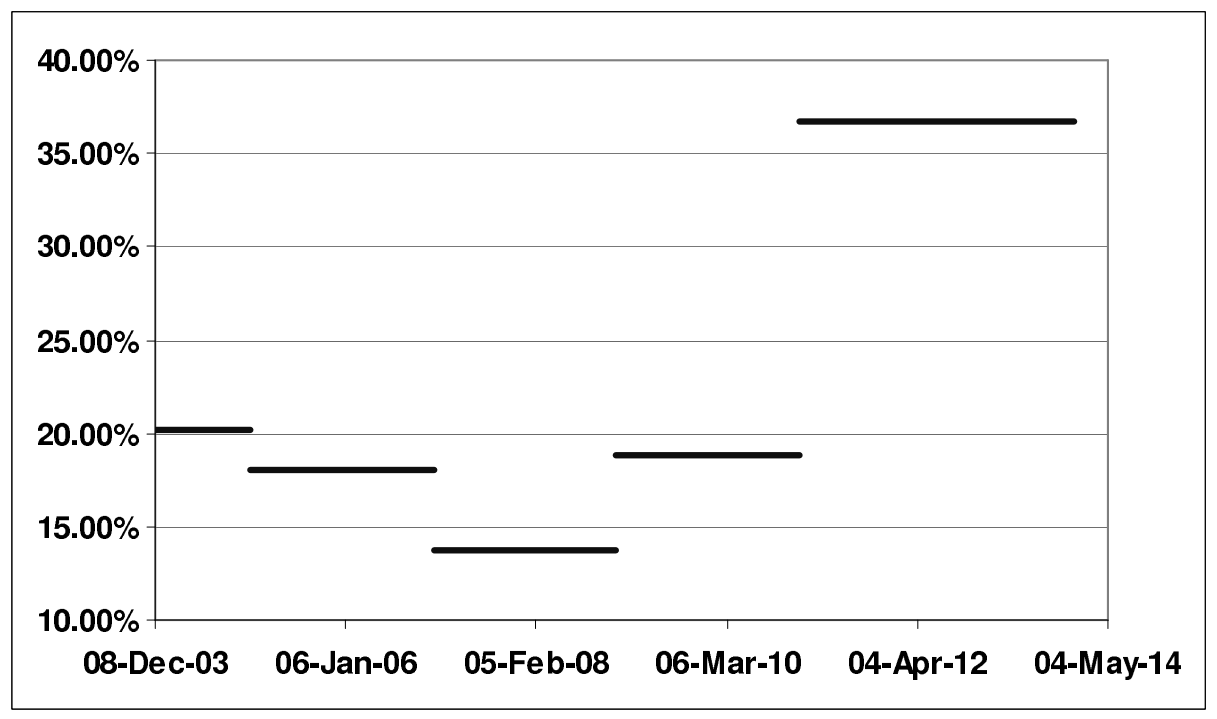

Figure 8: Volatility term structure $T \mapsto \sigma(T)$ on December 8th, 2003.

From the results (Tables 11 to [14), we see that in this second framework, the stripped volatilities are much higher than before, especially in proximity of the crisis, where they are suspiciously high for practical purposes and raise a number of questions on realism. However, this effect is essentially due to the low values of $H$ and not to $\beta$, as one could suspect. Indeed, in Table 15 we present the results of a calibration performed for the first date, with $\beta=0.08$ and $H$ computed with the "credit spread based" method, and it is immediately clear that the volatilities are no longer pathologically large and are nearly the same as in Table 7 . Anyway, the survival probabilities are not sensibly influenced by the method chosen to estimate $H$ when the CDS to be calibrated are fixed; this lack of sensitivity is mainly due to the peculiar role of $\beta$ and to the possibility to adjust its value, which in practice is 


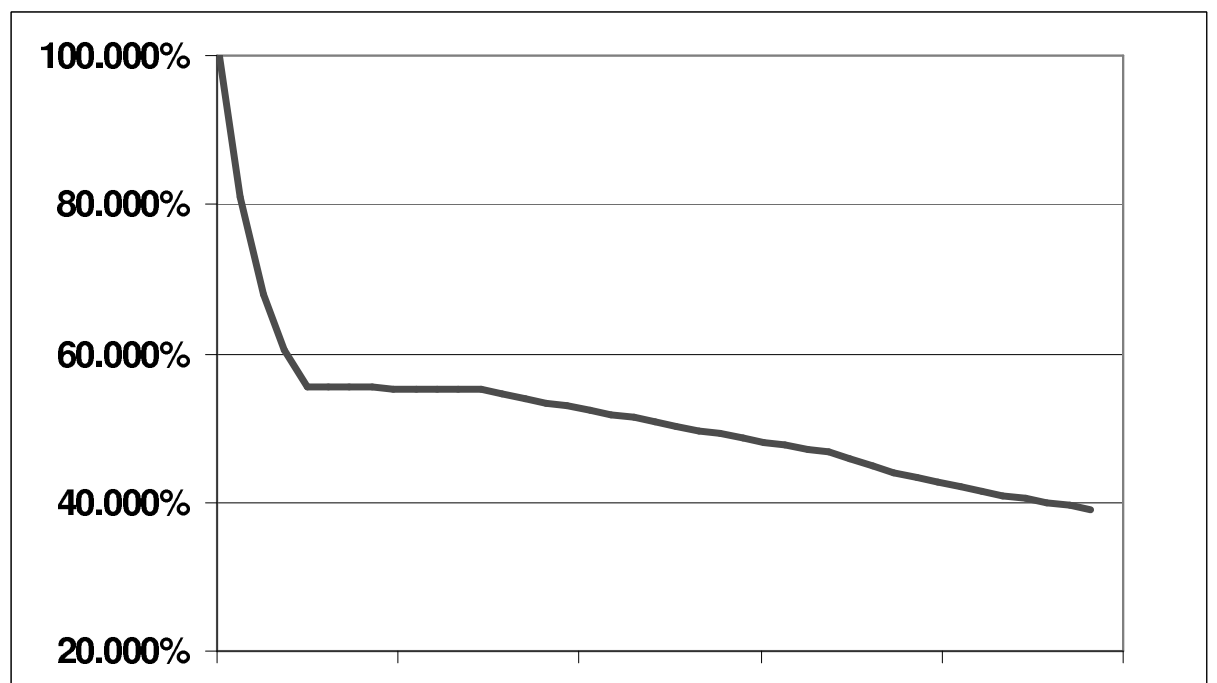

10-Dec-03 08-Jan-06 07-Feb-08 08-Mar-10 06-Apr-12 06-May-14

Figure 9: Survival probability on December 10th, 2003.

an important degree of freedom of the model.

Remark 4.2. (SBAT1P, Scenario Barrier version of the AT1P model) $A$ final remark on the possible use of random scenario-based barriers is in order. It is well known, see for example Bielecki and Rutkowski (2001), that in structural models with deterministic threshold barriers the default time is predictable. This is at times considered a drawback, especially in multi-name situations where one has to take into account contagion effects (Giesecke (2002)). What can help in this case is a random scenario-based default barrier. We may see that in our specific case the introduction of a random variable for the $H$ parameter, independent of the driving Brownian Motion $W$, induces through iterated conditioning an analytical formula for default probabilities and thus CDS's. This formula is based on a one dimensional integration of (18) in $H$ against the density of the random variable replacing the deterministic H. We call this model the SBAT1P (Scenario Barrier Analytically Tractable 1st Passage model) and we investigate its use in Brigo and Tarenghi (2005), where we use parameters in the distribution of $H$ to try and calibrate CDS prices. Scenarios on value of the firm volatilities can be employed as well (SVBAT1P model).

\section{A fundamental example: Pricing Counterparty Risk in Equity Swaps}

In this section we present an example of pricing with the calibrated structural model. This example concerns the valuation of an equity swap where we take into account counterparty risk, and is chosen to highlight one case where the calibrated structural model may be preferable to a reduced-form intensity model calibrated to the same market information. This is an illustration of a more general situation that typically occurs when one tries to price the counterparty risk in an equity payoff. We will see 
that it is possible to split the expectation of the payoff, and that the decomposition roughly involves the valuation of the same payoff without counterparty risk and the valuation of an option on the residual NPV of the considered payoff at the default time of the counterparty. Therefore including the counterparty risk adds an optionality level to the payoff.

Let us consider an equity swap payoff. Assume we are a company "A" entering a contract with company "B", our counterparty. The reference underlying equity is company "C". The contract, in its prototypical form, is built as follows. Companies "A" and "B" agree on a certain amount $K$ of stocks of a reference entity "C" (with price $S)$ to be taken as nominal $\left(N=K S_{0}\right)$. The contract starts in $T_{a}=0$ and has final maturity $T_{b}=T$. At $t=0$ there is no exchange of cash (alternatively, we can think that "B" delivers to "A" an amount $K$ of "C" stock and receives a cash amount equal to $K S_{0}$ ). At intermediate times "A" pays to "B" the dividend flows of the stocks (if any) in exchange for a periodic rate (for example a semi-annual LIBOR or EURIBOR rate $L$ ) plus a spread $X$. At final maturity $T=T_{b}$, "A" pays $K S_{T}$ to "B" (or gives back the amount $K$ of stocks) and receives a payment $K S_{0}$. This can be summarized as follows:

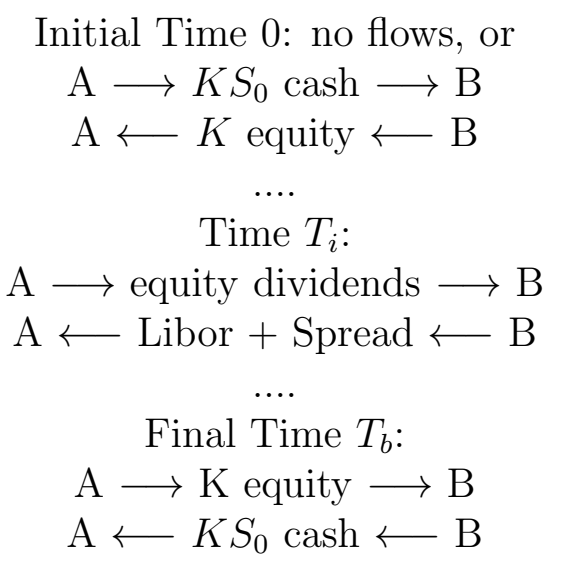

The price of this product can be derived using risk neutral valuation, and the (fair) spread is chosen in order to obtain a contract whose value at inception is zero. We ignore default of the underlying "C", thus assuming it has a much stronger credit quality than the counterparty "B". This can be the case for example when "C" is an equity index (Pignatelli (2004)). It can be proved that if we do not consider default risk for "B", the fair spread is identically equal to zero. But when taking into account counterparty default risk in the valuation the fair spread is no longer zero. In case an early default of the counterparty "B" occurs, the following happens. Let us call $\tau=\tau_{B}$ the default instant. Before $\tau$ everything is as before, but if $\tau \leq T$, the net present value (NPV) of the position at time $\tau$ is computed. If this NPV is negative for us, i.e. for "A", then its opposite is completely paid to "B" by us at time $\tau$ itself. On the contrary, if it is positive for "A", it is not received completely but only a recovery fraction $\mathrm{R}_{\mathrm{EC}}$ of that NPV is received by us. It is clear that to us ("A") the counterparty risk is a problem when the NPV is large and positive, since in case " $\mathrm{B}$ " defaults we receive only a fraction of it.

Analytically, the risk neutral expectation of the discounted payoff is $(L(S, T)$ is 
the simply compounded rate at time $S$ for maturity $T$ ):

$$
\begin{aligned}
\Pi_{E S}(0)= & \mathbb{E}_{0}\left\{\mathbf { 1 } _ { \{ \tau > T _ { b } \} } \left[-K \mathrm{NPV}_{\text {dividends }}^{0 \div T_{b}}(0)+K S_{0} \sum_{i=1}^{b} D\left(0, T_{i}\right) \alpha_{i}\left(L\left(T_{i-1}, T_{i}\right)\right.\right.\right. \\
& \left.+X)+D\left(0, T_{b}\right)\left(K S_{0}-K S_{T_{b}}\right)\right] \\
& +\mathbf{1}_{\left\{\tau \leq T_{b}\right\}}\left[-K \mathrm{NPV}_{\text {dividends }}^{0 \div \tau}(0)+K S_{0} \sum_{i=1}^{\beta(\tau)-1} D\left(0, T_{i}\right) \alpha_{i}\left(L\left(T_{i-1}, T_{i}\right)\right.\right. \\
& \left.\left.+X)+D(0, \tau)\left(\operatorname{REC}_{\mathrm{EC}}(\operatorname{NPV}(\tau))^{+}-(-\mathrm{NPV}(\tau))^{+}\right)\right]\right\}
\end{aligned}
$$

where

$$
\begin{aligned}
\operatorname{NPV}(\tau)= & \mathbb{E}_{\tau}\left\{-K \mathrm{NPV}_{\text {dividends }}^{\tau \dot{T_{b}}}(\tau)+K S_{0} \sum_{i=\beta(\tau)}^{b} D\left(\tau, T_{i}\right) \alpha_{i}\left(L\left(T_{i-1}, T_{i}\right)+X\right)\right. \\
& \left.+\left(K S_{0}-K S_{T_{b}}\right) D\left(\tau, T_{b}\right)\right\} .
\end{aligned}
$$

We denote by $\operatorname{NPV}_{\text {dividends }}^{s \div \text { t }}(u)$ the net present value of the dividend flows between $s$ and $t$ computed in $u$.

In the following we will prove the

Proposition 5.1. (Equity Return Swap price under Counterparty Risk). The fair price of the Equity Swap defined above, i.e. (22), can be simplified as follows:

$$
\Pi_{E S}(0)=K S_{0} X \sum_{i=1}^{b} \alpha_{i} P\left(0, T_{i}\right)-L_{G D} \mathbb{E}_{0}\left\{1_{\left\{\tau \leq T_{b}\right\}} P(0, \tau)(N P V(\tau))^{+}\right\}
$$

The first term is the equity swap price in a default-free world, whereas the second one is the optional price component due to counterparty risk.

Remark 5.2. (Different choice for the NPV). Let us focus for a second on the $N P V$ term in (23). In particular let us concentrate on the interval $\left[T_{\beta(\tau)-1}, T_{\beta(\tau)}\right)$ containing the default time $\tau$. If $\tau$ is strictly inside the interval, the LIBOR + spread resetting at the earlier $T_{\beta(\tau)-1}$ is paid for the whole interval.

A different formulation is the following. In case the default time is inside a certain interval, the $L I B O R+$ spread term for the part of this interval preceding default is immediately paid and we add the remaining part, to be paid at the end of the interval, discounted back from the end of the interval to the current (default) time. This amounts to let the summation in (23) start from $\beta(\tau)+1$ rather than from $\beta(\tau)$ and to add the following term in the NPV payoff:

$$
\left(L\left(T_{\beta(\tau)-1}, T_{\beta(\tau)}\right)+X\right)\left(\tau-T_{\beta(\tau)-1}\right)+\left(L\left(T_{\beta(\tau)-1}, T_{\beta(\tau)}\right)+X\right)\left(T_{\beta(\tau)}-\tau\right) D\left(\tau, T_{\beta(\tau)}\right)
$$

Formulation (23) reads like postponing the default $\tau$ to the first $T_{i}$ following $\tau$ and then discounting it back to the current (default) time. 
Both formulations make sense so contract specifications are important in order to price this kind of products. Analytical pricing formulas with our choice result to be more tractable than with the alternative choice presented in this remark, where small accruing terms appear. Such accruing terms are very small in general and can be ignored.

If we try and find the above price by computing the expectation through a Monte Carlo simulation, we have to simulate both the behavior of the equity "C" underlying the swap, which we call $S_{t}=S_{t}^{C}$, and the default of the counterparty "B". In particular we need to know exactly $\tau=\tau_{B}$. Obviously the correlation between "B" and "C" could have a relevant impact on the contract value. Here the structural model can be helpful: Suppose to calibrate the underlying process $V$ to CDS's for name "B", finding the appropriate default barrier and volatilities according to the procedure outlined earlier in this paper with the AT1P model. We could set a correlation between the processes $V_{t}^{B}$ for "B" and $S_{t}$ for "C", derived for example through historical estimation directly based on equity returns, and simulate the joint evolution of $\left[V_{t}^{B}, S_{t}\right]$. As a proxy of the correlation between these two quantities we may consider the correlation between $S_{t}^{B}$ and $S_{t}^{C}$, i.e. between equities.

Remark 5.3. (Using reduced form models?) At this point it may be interesting to consider the alternatives offered by an intensity reduced form model. If one takes a deterministic intensity, since Poisson processes and Brownian Motions defined on the same space are independent, there is no way to introduce a correlation between the default event for " $B$ " and the value of the equity " $C$ ". To take into account this correlation we may have to move to stochastic intensity. However, correlating the stochastic intensity of " $B$ " to the equity of " $C$ " may lead to a poorly tractable model, even under tractable intensities as in Brigo and Alfonsi (2005), and the amount of correlation induced in this way may be too small for practical purposes. Furthermore, historical estimation of the correlation between the instantaneous intensity and the equity of " $C$ " may pose some problems that the structural model avoids by definition.

Now let us go more into details in analyzing the payoff. For simplicity, we assume deterministic dividends and rates, so that we can substitute the discount factors $D(t, T)$ with zero coupon bond prices $P(t, T)$ and move them outside the expectations. Moreover we recall that $L\left(T_{i-1}, T_{i}\right)=\left(P\left(0, T_{i-1}\right) / P\left(0, T_{i}\right)-1\right) / \alpha_{i}$ and, as a consequence, $\sum_{i=A+1}^{B} P\left(0, T_{i}\right) \alpha_{i} L\left(T_{i-1}, T_{i}\right)=P\left(0, T_{A}\right)-P\left(0, T_{B}\right)$. Finally, we set $\mathrm{R}_{\mathrm{EC}}=1-\mathrm{L}_{\mathrm{GD}}$, consistently with CDS notation, and notice that

$$
\operatorname{ReC}_{\mathrm{EC}}(\operatorname{NPV}(\tau))^{+}-(-\mathrm{NPV}(\tau))^{+}=\operatorname{NPV}(\tau)-\mathrm{LGD}(\mathrm{NPV}(\tau))^{+}
$$

We can rewrite the discounted NPV as

$$
\begin{aligned}
P(0, \tau) \mathrm{NPV}(\tau)= & -K \mathrm{NPV}_{\text {dividends }}^{\tau \dot{*} T_{b}}(0)+K S_{0} \sum_{i=\beta(\tau)}^{b} P\left(0, T_{i}\right) \alpha_{i}\left(L\left(T_{i-1}, T_{i}\right)+X\right) \\
& +K S_{0} P\left(0, T_{b}\right)-K \mathbb{E}_{\tau}\left\{S_{T_{b}}\right\} P\left(0, T_{b}\right) .
\end{aligned}
$$


Substituting (24) and (25) in (22) we obtain:

$$
\begin{aligned}
& \Pi_{E S}(0)=\mathbb{E}_{0}\left\{\mathbf { 1 } _ { \{ \tau > T _ { b } \} } \left[-K \mathrm{NPV}_{\text {dividends }}^{0 \div T_{b}}(0)+K S_{0} \sum_{i=1}^{b} P\left(0, T_{i}\right) \alpha_{i}\left(L\left(T_{i-1}, T_{i}\right)+X\right)\right.\right. \\
& \left.+P\left(0, T_{b}\right)\left(K S_{0}-K S_{T_{b}}\right)\right] \\
& +\mathbf{1}_{\left\{\tau \leq T_{b}\right\}}\left[-K \mathrm{NPV}_{\text {dividends }}^{0 \div \tau}(0)+K S_{0} \sum_{i=1}^{\beta(\tau)-1} P\left(0, T_{i}\right) \alpha_{i}\left(L\left(T_{i-1}, T_{i}\right)+X\right)\right. \\
& \left.\left.+P(0, \tau) \operatorname{NPV}(\tau)-\mathrm{LGD}_{\mathrm{GD}} P(0, \tau)(\mathrm{NPV}(\tau))^{+}\right]\right\}= \\
& =\mathbb{E}_{0}\left\{\mathbf { 1 } _ { \{ \tau > T _ { b } \} } \left[-K \mathrm{NPV}_{\text {dividends }}^{0 \div T_{b}}(0)+K S_{0} \sum_{i=1}^{b} P\left(0, T_{i}\right) \alpha_{i}\left(L\left(T_{i-1}, T_{i}\right)+X\right)\right.\right. \\
& \left.+P\left(0, T_{b}\right) K S_{0}-P\left(0, T_{b}\right) K S_{T_{b}}\right] \\
& +\mathbf{1}_{\left\{\tau \leq T_{b}\right\}}\left[-K \mathrm{NPV}_{\text {dividends }}^{0 \div T_{b}}(0)+K S_{0} \sum_{i=1}^{b} P\left(0, T_{i}\right) \alpha_{i}\left(L\left(T_{i-1}, T_{i}\right)+X\right)\right. \\
& \left.\left.+P\left(0, T_{b}\right) K S_{0}-P\left(0, T_{b}\right) K \mathbb{E}_{\tau}\left\{S_{T_{b}}\right\}-\mathrm{LGD}_{\mathrm{GD}} P(0, \tau)(\mathrm{NPV}(\tau))^{+}\right]\right\}= \\
& =-K \mathrm{NPV}_{\text {dividends }}^{0 \div T_{b}}(0)+K S_{0} \sum_{i=1}^{b} P\left(0, T_{i}\right) \alpha_{i}\left(L\left(T_{i-1}, T_{i}\right)+X\right) \\
& +P\left(0, T_{b}\right) K S_{0}-P\left(0, T_{b}\right) K \mathbb{E}_{0}\left\{\mathbf{1}_{\left\{\tau>T_{b}\right\}} S_{T_{b}}\right\}-P\left(0, T_{b}\right) K \mathbb{E}_{0}\left\{\mathbf{1}_{\left\{\tau \leq T_{b}\right\}} \mathbb{E}_{\tau}\left\{S_{T_{b}}\right\}\right\} \\
& -\mathrm{L}_{\mathrm{GD}} \mathbb{E}_{0}\left\{\mathbf{1}_{\left\{\tau \leq T_{b}\right\}} P(0, \tau)(\mathrm{NPV}(\tau))^{+}\right\}= \\
& =-K \mathrm{NPV}_{\text {dividends }}^{0 \div T_{b}}(0)+K S_{0}+K S_{0} X \sum_{i=1}^{b} \alpha_{i} P\left(0, T_{i}\right) \\
& -P\left(0, T_{b}\right) K \mathbb{E}_{0}\left\{\mathbf{1}_{\left\{\tau>T_{b}\right\}} S_{T_{b}}+\mathbf{1}_{\left\{\tau \leq T_{b}\right\}} \mathbb{E}_{\tau}\left\{S_{T_{b}}\right\}\right\} \\
& -\mathrm{L}_{\mathrm{GD}} \mathbb{E}_{0}\left\{\mathbf{1}_{\left\{\tau \leq T_{b}\right\}} P(0, \tau)(\mathrm{NPV}(\tau))^{+}\right\} \text {. }
\end{aligned}
$$

Now, observing that

$$
\begin{aligned}
\mathbb{E}_{0}\left\{\mathbf{1}_{\left\{\tau>T_{b}\right\}} S_{T_{b}}+\mathbf{1}_{\left\{\tau \leq T_{b}\right\}} \mathbb{E}_{\tau}\left\{S_{T_{b}}\right\}\right\} & =\mathbb{E}_{0}\left\{\mathbf{1}_{\left\{\tau>T_{b}\right\}} S_{T_{b}}+\left(1-\mathbf{1}_{\left\{\tau>T_{b}\right\}}\right) \mathbb{E}_{\tau}\left\{S_{T_{b}}\right\}\right\}= \\
& =\mathbb{E}_{0}\left\{\mathbb{E}_{\tau}\left\{S_{T_{b}}\right\}+\mathbf{1}_{\left\{\tau>T_{b}\right\}}\left(S_{T_{b}}-\mathbb{E}_{\tau}\left\{S_{T_{b}}\right\}\right)\right\}= \\
& =\mathbb{E}_{0}\left\{\mathbb{E}_{\tau}\left\{S_{T_{b}}\right\}\right\}+\mathbb{E}_{0}\left\{\mathbf{1}_{\left\{\tau>T_{b}\right\}}\left(S_{T_{b}}-S_{T_{b}}\right)\right\}= \\
& =\mathbb{E}_{0}\left\{S_{T_{b}}\right\}=\frac{S_{0}}{P\left(0, T_{b}\right)}-\frac{\mathrm{NPV}_{\text {dividends }}(0)}{P\left(0, T_{b}\right)}
\end{aligned}
$$


the expected payoff (26) becomes

$$
\begin{aligned}
\Pi_{E S}(0)= & -K \mathrm{NPV}_{\text {dividends }}^{0 \div T_{b}}(0)+K S_{0}+K S_{0} X \sum_{i=1}^{b} \alpha_{i} P\left(0, T_{i}\right) \\
& -K P\left(0, T_{b}\right)\left(\frac{S_{0}}{P\left(0, T_{b}\right)}-\frac{\mathrm{NPV}_{\text {dividends }}^{0 \div T_{b}}(0)}{P\left(0, T_{b}\right)}\right) \\
& -\mathrm{LGD}_{\mathrm{GD}} \mathbb{E}_{0}\left\{\mathbf{1}_{\{\tau \leq T\}} P(0, \tau)(\mathrm{NPV}(\tau))^{+}\right\}= \\
= & K S_{0} X \sum_{i=1}^{b} \alpha_{i} P\left(0, T_{i}\right)-\mathrm{LGD}_{\mathbb{G}} \mathbb{E}_{0}\left\{\mathbf{1}_{\left\{\tau \leq T_{b}\right\}} P(0, \tau)(\mathrm{NPV}(\tau))^{+}\right\} .
\end{aligned}
$$

which proves our proposition above. As noticed in the proposition, the first term in the last expression in (28) is $\prod_{E S}^{\text {DEFREE }}(0)$, i.e. the payoff of the Equity Swap in a default free ("defree") world. Indeed, as stated before, in a default free market the fair spread would be zero, so that if we add a nonzero spread $X$ the value of the equity swap in a default free world is simply the NPV of the future spread cash flows.

The second term is very important if we allow counterparty "B" to default. As it is non-positive, to have a fair value for $\Pi_{E S}(0)$ we need a non-negative spread $X$.

At this point it may be worth noticing that the above decomposition is rather general. When we include counterparty risk into the valuation, we always obtain a decomposition

$$
\text { Price }=\text { PricedefaultFree - (1-recovery) CallOptionon residual NPV }
$$

The call option has strike 0 . As we have observed earlier, the counterparty risk introduces, among other features, a further optionality level into the valuation.

Going back to our equity swap, now it is possible to run the Monte Carlo simulation, looking for the spread $X$ that makes the contract fair. The simulation itself is simpler when taking into account the following computation included in the discounted NPV:

$$
P\left(\tau, T_{b}\right) \mathbb{E}_{\tau}\left\{S_{T_{b}}\right\}=S_{\tau}-\mathrm{NPV}_{\text {dividends }}^{\tau \div T_{b}}(\tau)
$$

so that we have

$$
\begin{aligned}
P(0, \tau) \mathrm{NPV}(\tau)= & K S_{0} \sum_{i=\beta(\tau)}^{b} P\left(0, T_{i}\right) \alpha_{i}\left(L\left(T_{i-1}, T_{i}\right)+X\right)+K S_{0} P\left(0, T_{b}\right) \\
& -K P(0, \tau) S_{\tau}= \\
= & K S_{0} \sum_{i=\beta(\tau)}^{b} P\left(0, T_{i}\right) \alpha_{i} X+K S_{0} P\left(0, T_{\beta(\tau)-1}\right) \\
& -K P(0, \tau) S_{\tau} .
\end{aligned}
$$

The reformulation of the original expected payoff (22) as in (28) presents an important advantage in terms of numerical simulation. In fact in (22) we have a global expectation, hence we have to simulate the exact payoff for each path. In (28), with many simplifications, we have isolated a part of the expected payoff out of the 
main expectation. This isolated part has an expected value that we have been able to calculate, so that it does not have to be simulated. Simulating only the residual part is helpful because now the variance of the part of the payoff that has been computed analytically is no longer affecting the standard error of our Monte Carlo simulation. The standard error is indeed much lower when simulating (28) instead of (22)). The expected value we computed analytically above involves terms in $S_{T_{b}}$ which would add a lot of variance to the final payoff. In (28) the only $S_{T_{b}}$ term left is in the optional NPV part.

We performed some simulations under different assumptions on the correlation between "B" and "C". We considered five cases: $\rho=-1, \rho=-0.2, \rho=0, \rho=0.5$ and $\rho=1$. In Table 6 we present the results of the simulation, together with the error given by one standard deviation (Monte Carlo standard error). For counterparty "B" we used the same CDS rates of the company analyzed in Section 3. For the reference stock "C" we used a hypothetical stock with initial price $S_{0}=20$, volatility $\sigma=20 \%$ and constant dividend yield $q=0.80 \%$. The contract has maturity $T=5 y$ and the settlement of the LIBOR rate has a semi-annual frequency. Finally, we included a recovery rate $R_{\mathrm{EC}}=40 \%$. The starting date is the same we used for the calibration, i.e. March 10th, 2004. Since the reference number of stocks $K$ is just a constant multiplying the whole payoff, without losing generality we set it equal to one.

In order to reduce the errors of the simulations, we have adopted a variance reduction technique using the default indicator (whose expected value is the known default probability) as a control variate. In particular we have used the default indicator $1_{\{\tau<T\}}$ at the maturity $T$ of the contract, which has a large correlation with the final payoff. Even so, a large number of scenarios are needed to obtain errors with a lower order of magnitude than $X$. In our simulations we have used $N=2000000$.

We notice that $X$ increases together with $\rho$. This fact can be explained in the following way. Let us consider the case of positive correlation between " $\mathrm{B}$ " and " $\mathrm{C}$ ": This means that, in general, if the firm value for " $\mathrm{B}$ " increases, moving away from the default barrier, also the stock price for "C" tends to increase due to the positive correlation. Both processes will then have high values. Instead, again under positive correlation, if $V_{t}^{B}$ lowers towards the default barrier, also $S_{t}^{C}$ will tend to do so, going possibly below the initial value $S_{0}$. In this case $N P V(\tau)$ has a large probability to be positive (see (30) ), so that one needs a large $X$ to balance it, as is clear when looking at the final payoff (28). On the contrary, for negative correlation, the same reasoning can be applied, but now if $V_{t}^{B}$ lowers and tends to the default barrier, in general $S_{t}^{C}$ will tend to move in the opposite direction and the corresponding $N P V(\tau)$ will probably be negative, or, if positive, not very large. Hence the "balancing" spread $X$ we need will be quite small.

The simulation error depends on $\rho$ too, and in particular it is influenced by two main effects. The first one is the same as before. Indeed, a positive correlation means high probability of positive NPV in case of default. As the default probability of "B" does not depend on $\rho$, it is clear that a positive correlation leads to evaluate the expectation of a random variable which has a high probability of being different from zero (and in particular positive), while a negative correlation leads to the expectation of a variable which is almost always zero, leading to a lower Monte Carlo error. The other effect is due to the impact of $\rho$ on the correlation between the payoff variate and the control variate. It happens that this correlation is decreasing in $\rho$ : It is 


\begin{tabular}{|c|c|c|c|}
\hline$\rho$ & $\mathrm{X}$ & ES payoff & MC error \\
\hline-1 & 0 & 0 & $0^{*}$ \\
-0.2 & 2.45 & -0.02 & 1.71 \\
0 & 4.87 & -0.90 & 2.32 \\
0.5 & 14.2 & -0.53 & 2.71 \\
1 & 24.4 & -0.34 & 0.72 \\
\hline
\end{tabular}

Table 6: Fair spread $X$ (in basis points) of the Equity Swap in five different correlation cases, $S_{0}=20$. We also report the value of the average of the simulated payoff (times 10000) across the 2000000 scenarios and its standard error, for the chosen $X$, thus showing that $X$ is indeed fair in practice, since all the averages and MC windows are close to zero. $X$ 's have been found by iterating the MC simulation for different values of $X$ until the payoff was found to be sufficiently small. The MC error for $\rho=-1$ (marked with an asterisk) is null since in each simulated scenario the simulated term has been found to be identically zero (i.e. the NPV was always negative).

nearly -1 when $\rho=1$, and then increases with lower values of $\rho$ (it is about -0.4 for $\rho=-0.2)$.

As a final remark we notice that the spread $X$ corresponding to $\rho=-1$ is identically equal to zero. The reason is quite simple: When we have maximum negative correlation between the counterparty "B" and the reference entity "C", the NPV in (28) is negative in all the simulated scenarios involving default, so that the related term in the price of the equity swap is always zero. We are then left only with the fixed term and this is null only when $X$ is zero. All this reasoning also explains why the error of the simulation, marked with an asterisk, is zero.

\section{Conclusions}

In general the link between default probabilities and credit spreads is best described by intensity models. The credit spread to be added to the risk free rate represents a good measure of a bond credit risk for example. Yet, intensity models present some drawbacks: They do not link the default event to the economy but rather to an exogenous jump process whose jump component remains unexplained from an economic point of view. Moreover, when dealing with default correlation, a copula function must be introduced between the jump processes thresholds in a way that has no clear immediate relation with equity correlation, the only source of correlation that can be used for practical purposes.

In this paper we introduced an analytically tractable structural model that allows for a solution to the above points. In this model the default has an economic cause, in that it is caused by the value of the firm hitting the safety barrier value, and all quantities are basic market observables. Also, when dealing with multi-name products, the model allows for the introduction of the correlation in a very natural way, by simply correlating shocks in the different values of the firm equities by means of equity correlation.

We showed how to calibrate the model parameters to actual market data: Starting from CDS quotes, we calibrated the value of the firm volatilities and found the 
barrier triggering the default that was consistent with CDS quotes and also leading to analytical tractability. We also explained the analogies with barrier option pricing, in particular the case with time dependent parameters. We hinted at the possible use of a scenario based random barrier, as in Brigo and Tarenghi (2005).

As a practical example, we also applied the model to a concrete case, showing how it can describe the proximity of default when time changes and the market quotes for the CDS's reflect increasing credit deterioration. When the market detects a company crisis, it responds with high CDS quotes and this translates into high default probabilities, i.e. high probabilities for the underlying process to hit the safety barrier, that in turn translate in high calibrated volatilities for the firm value dynamics. We also took advantage of the numerical examples to further explain and interpret a few model parameters whose role needed further investigation.

The calibrated model is useful for pricing more sophisticated derivatives depending on default. We gave the fundamental example of equity swaps pricing under counterparty risk.

\section{Appendix: Results of the case study calibrations and market data}

\subsection{Credit spread based barrier parameter $H$}

Here we present the numerical results of the calibration relative to the case study discussed in Section 4. For each date we present the spot price of the equity with its historical volatility, the rates and the recovery rates of the (running) CDS's (quarterly paid), the outputs of the calibration. For all cases we used $\beta=0.5$. $H$ is computed using the credit spread based method explained in Section 3 .

- September 10th, 2003.

Stock Price $=2.898$ euros, volatility $=5 \%$, recovery rate $=40 \%$. The barrier parameter is $H=0.8977$ (re-scaled $V_{0}=1$ ).

\begin{tabular}{|c|c|c|c|}
\hline CDS maturity & Rate (bps) & Volatility nodes & Survival \\
\hline 10-sep-03 & - & $5.012 \%$ & $100.000 \%$ \\
$1 \mathrm{y}$ & 192.5 & $5.012 \%$ & $96.673 \%$ \\
$3 \mathrm{y}$ & 215 & $3.103 \%$ & $89.524 \%$ \\
$5 \mathrm{y}$ & 225 & $3.178 \%$ & $82.471 \%$ \\
$7 \mathrm{y}$ & 235 & $3.551 \%$ & $75.375 \%$ \\
$10 \mathrm{y}$ & 235 & $3.658 \%$ & $66.998 \%$ \\
\hline
\end{tabular}

Table 7: Outputs of the calibration on September 10th, 2003. Here $\beta=0.5$ and $H=$ 0.8977 .

- November 28th, 2003.

Stock Price $=2.297$ euros, volatility $=14 \%$, recovery rate $=40 \%$. The barrier parameter is $H=0.8052$ (re-scaled $V_{0}=1$ ). 


\begin{tabular}{|c|c|c|c|}
\hline CDS maturity & Rate (bps) & Volatility nodes & Survival \\
\hline 28-nov-03 & - & $14.081 \%$ & $100.000 \%$ \\
1y & 725 & $14.081 \%$ & $87.620 \%$ \\
$3 \mathrm{y}$ & 630 & $10.800 \%$ & $72.529 \%$ \\
$5 \mathrm{y}$ & 570 & $11.489 \%$ & $62.561 \%$ \\
$7 \mathrm{y}$ & 570 & $16.235 \%$ & $51.810 \%$ \\
$10 \mathrm{y}$ & 570 & $22.793 \%$ & $39.217 \%$ \\
\hline
\end{tabular}

Table 8: Outputs of the calibration on November 28th, 2003. Here $\beta=0.5$ and $H=0.8052$.

- December 8th, 2003.

Stock Price $=2.237$ euros, volatility $=20 \%$, recovery rate $=25 \%$. The barrier parameter is $H=0.7730$ (re-scaled $V_{0}=1$ ).

\begin{tabular}{|c|c|c|c|}
\hline CDS maturity & Rate (bps) & Volatility nodes & Survival \\
\hline 8-dec-03 & - & $20.197 \%$ & $100.000 \%$ \\
1y & 1450 & $20.197 \%$ & $81.289 \%$ \\
3y & 1200 & $17.972 \%$ & $62.065 \%$ \\
$5 \mathrm{y}$ & 940 & $13.685 \%$ & $56.263 \%$ \\
$7 \mathrm{y}$ & 850 & $18.771 \%$ & $49.386 \%$ \\
10y & 850 & $36.661 \%$ & $35.626 \%$ \\
\hline
\end{tabular}

Table 9: Outputs of the calibration on December 8th, 2003. Here $\beta=0.5$ and $H=0.7730$.

- December 10th, 2003.

Stock Price $=2.237$ euros, volatility $=50 \%$, recovery rate $=15 \%$. The barrier parameter is $H=0.7253$ (re-scaled $V_{0}=1$ ).

\begin{tabular}{|c|c|c|c|}
\hline CDS maturity & Rate (bps) & Volatility nodes & Survival \\
\hline 10-dec-03 & - & $50.000 \%$ & $100.000 \%$ \\
$1 \mathrm{y}$ & 5050 & $50.000 \%$ & $55.452 \%$ \\
$3 \mathrm{y}$ & 2100 & $4.325 \%$ & $55.208 \%$ \\
$5 \mathrm{y}$ & 1500 & $19.950 \%$ & $50.910 \%$ \\
$7 \mathrm{y}$ & 1250 & $24.063 \%$ & $46.705 \%$ \\
$10 \mathrm{y}$ & 1100 & $37.422 \%$ & $39.121 \%$ \\
\hline
\end{tabular}

Table 10: Outputs of the calibration on December 10th, 2003. Here $\beta=0.5$ and $H=$ 0.7253 .

Remark 7.1. In this last case the calibration algorithm for the deterministic intensity model could not achieve a solution, so it was not possible to extract the suitable value for the "credit spread based" $H$. In such cases we can proceed in an alternative way. Let $\bar{\sigma}$ be the equity volatility for the first maturity that is given by the equity market.

a. Choose a first guess $H^{(1)}$ for $H$. Set $i=1$; 
b. Calibrate the structural model to the first maturity CDS market quote through the parameter $\sigma$ when the barrier is set to $H^{(i)}$; Let $\sigma^{(i)}$ be the calibrated volatility.

c. Compute the first maturity default probability with the structural model characterized by $H^{(i)}$ and $\sigma^{(i)}$.

d. Solve in $H$ the equation where we equate the default probability computed in "c" above to the default probability of a structural model characterized by $H$ and $\bar{\sigma}$; Set $H^{(i+1)}$ to the found value of $H$; Replace $i$ by $i+1$ and restart from "b".

Typically, after a number of iterations, $\sigma^{(i)}$ will be very close to $\bar{\sigma}$ and we may set $H$ to the corresponding value $H^{(i)}$.

\subsection{Excursion/protection analogy barrier parameter $H$}

Here we present the results of the calibration performed using the excursion protection analogy to estimate $H$, i.e. $H / V_{0}=\mathrm{R}_{\mathrm{EC}}$. Now $\beta=0.08$ and the other parameters are the same as before.

- September 10th, 2003.

The barrier parameter is $H=\mathrm{R}_{\mathrm{EC}}=0.4$ (re-scaled $V_{0}=1$ ).

\begin{tabular}{|c|c|c|c|}
\hline CDS maturity & Rate (bps) & Volatility nodes & Survival \\
\hline 10-sep-03 & - & $42.713 \%$ & $100.000 \%$ \\
1y & 192.5 & $42.713 \%$ & $96.674 \%$ \\
$3 \mathrm{y}$ & 215 & $26.588 \%$ & $89.524 \%$ \\
$5 \mathrm{y}$ & 225 & $27.343 \%$ & $82.473 \%$ \\
$7 \mathrm{y}$ & 235 & $30.686 \%$ & $75.377 \%$ \\
10y & 235 & $31.778 \%$ & $67.002 \%$ \\
\hline
\end{tabular}

Table 11: Outputs of the calibration on September 10th, 2003. Here $\beta=0.08$ and $H=0.4$.

- November 28th, 2003.

The barrier parameter is $H=\mathrm{R}_{\mathrm{EC}}=0.4$ (re-scaled $V_{0}=1$ ).

\begin{tabular}{|c|c|c|c|}
\hline CDS maturity & Rate (bps) & Volatility nodes & Survival \\
\hline $28-$ nov-03 & - & $58.808 \%$ & $100.000 \%$ \\
$1 \mathrm{y}$ & 725 & $58.808 \%$ & $87.613 \%$ \\
$3 \mathrm{y}$ & 630 & $44.225 \%$ & $72.512 \%$ \\
$5 \mathrm{y}$ & 570 & $46.237 \%$ & $62.541 \%$ \\
$7 \mathrm{y}$ & 570 & $63.898 \%$ & $51.780 \%$ \\
$10 \mathrm{y}$ & 570 & $85.758 \%$ & $39.146 \%$ \\
\hline
\end{tabular}

Table 12: Outputs of the calibration on November 28th, 2003. Here $\beta=0.08$ and $H=0.4$. 
- December 8th, 2003.

The barrier parameter is $H=\mathrm{R}_{\mathrm{EC}}=0.25$ (re-scaled $V_{0}=1$ ).

\begin{tabular}{|c|c|c|c|}
\hline CDS maturity & Rate (bps) & Volatility nodes & Survival \\
\hline 8-dec-03 & - & $107.846 \%$ & $100.000 \%$ \\
1y & 1450 & $107.846 \%$ & $81.277 \%$ \\
3y & 1200 & $94.506 \%$ & $62.041 \%$ \\
$5 \mathrm{y}$ & 940 & $71.046 \%$ & $56.246 \%$ \\
$7 \mathrm{y}$ & 850 & $96.431 \%$ & $49.369 \%$ \\
$10 \mathrm{y}$ & 850 & $181.575 \%$ & $35.559 \%$ \\
\hline
\end{tabular}

Table 13: Outputs of the calibration on December 8th, 2003. Here $\beta=0.08$ and $H=0.25$.

- December 10th, 2003.

The barrier parameter is $H=\mathrm{R}_{\mathrm{EC}}=0.15$ (re-scaled $V_{0}=1$ ).

\begin{tabular}{|c|c|c|c|}
\hline CDS maturity & Rate (bps) & Volatility nodes & Survival \\
\hline 10-dec-03 & - & $292.060 \%$ & $100.000 \%$ \\
$1 \mathrm{y}$ & 5050 & $292.060 \%$ & $55.381 \%$ \\
$3 \mathrm{y}$ & 2100 & $21.603 \%$ & $55.198 \%$ \\
$5 \mathrm{y}$ & 1500 & $114.713 \%$ & $50.904 \%$ \\
$7 \mathrm{y}$ & 1250 & $137.488 \%$ & $46.700 \%$ \\
$10 \mathrm{y}$ & 1100 & $210.474 \%$ & $39.104 \%$ \\
\hline
\end{tabular}

Table 14: Outputs of the calibration on December 10th, 2003. Here $\beta=0.08$ and $H=0.15$.

Finally, we present the calibration performed for the first date (September 10th, 2003) with $\beta=0.08$ and $H$ estimated by means of the credit spread method. Comparing the values in Table 15 with those in Table 7 we see that the volatilities are nearly the same, and this holds in general, at least when the volatilities are not too high. Here the barrier parameter is $H=0.8969$ (re-scaled $V_{0}=1$ ).

\begin{tabular}{|c|c|c|c|}
\hline CDS maturity & Rate (bps) & Volatility nodes & Survival \\
\hline 10-sep-03 & - & $5.012 \%$ & $100.000 \%$ \\
$1 \mathrm{y}$ & 192.5 & $5.012 \%$ & $96.673 \%$ \\
$3 \mathrm{y}$ & 215 & $3.064 \%$ & $89.522 \%$ \\
$5 \mathrm{y}$ & 225 & $3.110 \%$ & $82.468 \%$ \\
$7 \mathrm{y}$ & 235 & $3.445 \%$ & $75.369 \%$ \\
$10 \mathrm{y}$ & 235 & $3.508 \%$ & $66.988 \%$ \\
\hline
\end{tabular}

Table 15: Outputs of the calibration on September 10th, 2003. Here $\beta=0.08$ and $H=$ 0.8969 . 


\subsection{Discount Curves}

In the following we report the Discount Curves that have been used in the simulations.

- September 10th, 2003.

\begin{tabular}{|c|c||c|c||c|c|}
\hline Date & Discount & Date & Discount & Date & Discount \\
\hline Sep 10th, 03 & 1.00000 & Jun 14th, 04 & 0.98320 & Sep 12th, 11 & 0.71837 \\
Sep 11th, 03 & 0.99994 & Sep 13th, 04 & 0.97721 & Sep 12th, 12 & 0.68171 \\
Sep 15th, 03 & 0.99971 & Sep 12th, 05 & 0.94740 & Sep 12th, 13 & 0.64624 \\
Sep 19th, 03 & 0.99948 & Sep 12th, 06 & 0.91195 & Sep 12th, 14 & 0.61204 \\
Oct 13th, 03 & 0.99805 & Sep 12th, 07 & 0.87373 & Sep 14th, 15 & 0.58032 \\
Nov 12th, 03 & 0.99627 & Sep 12th, 08 & 0.83501 & Sep 12th, 16 & 0.54942 \\
Dec 12th, 03 & 0.99447 & Sep 14th, 09 & 0.79557 & Sep 12th, 17 & 0.51882 \\
Mar 12th, 04 & 0.98896 & Sep 13th, 10 & 0.75666 & Sep 12th, 18 & 0.48937 \\
\hline
\end{tabular}

Table 16: Discount curve on September 10th, 2003.

- November 28th, 2003.

\begin{tabular}{|c|c||c|c||c|c|}
\hline Date & Discount & Date & Discount & Date & Discount \\
\hline Nov 28th, 03 & 1.00000 & Sep 02nd, 04 & 0.98200 & Dec 02nd, 11 & 0.70648 \\
Dec 01st, 03 & 0.99983 & Dec 02nd, 04 & 0.97508 & Dec 03rd, 12 & 0.66950 \\
Dec 03rd, 03 & 0.99971 & Dec 02nd, 05 & 0.94202 & Dec 02nd, 13 & 0.63449 \\
Dec 09th, 03 & 0.99936 & Dec 04th, 06 & 0.90424 & Dec 02nd, 14 & 0.60081 \\
Jan 02nd, 04 & 0.99792 & Dec 03rd, 07 & 0.86449 & Dec 02nd, 15 & 0.56852 \\
Feb 02nd, 04 & 0.99607 & Dec 02nd, 08 & 0.82431 & Dec 02nd, 16 & 0.53760 \\
Mar 02nd, 04 & 0.99435 & Dec 02nd, 09 & 0.78427 & Dec 04th, 17 & 0.50786 \\
Jun 02nd, 04 & 0.98849 & Dec 02nd, 10 & 0.74490 & Dec 03rd, 18 & 0.47965 \\
\hline
\end{tabular}

Table 17: Discount curve on November 28th, 2003.

- December 8th, 2003.

\begin{tabular}{|c|c||c|c||c|c|}
\hline Date & Discount & Date & Discount & Date & Discount \\
\hline Dec 08th, 03 & 1.00000 & Sep 10th, 04 & 0.98271 & Dec 12th, 11 & 0.71134 \\
Dec 09th, 03 & 0.99994 & Dec 10th, 04 & 0.97612 & Dec 10th, 12 & 0.67460 \\
Dec 11th, 03 & 0.99983 & Dec 12th, 05 & 0.94456 & Dec 10th, 13 & 0.63960 \\
Dec 17th, 03 & 0.99949 & Dec 11th, 06 & 0.90787 & Dec 10th, 14 & 0.60587 \\
Jan 12th, 04 & 0.99793 & Dec 10th, 07 & 0.86880 & Dec 10th, 15 & 0.57341 \\
Feb 10th, 04 & 0.99620 & Dec 10th, 08 & 0.82911 & Dec 12th, 16 & 0.54209 \\
Mar 10th, 04 & 0.99448 & Dec 10th, 09 & 0.78937 & Dec 11th, 17 & 0.51229 \\
Jun 10th, 04 & 0.98882 & Dec 10th, 10 & 0.74996 & Dec 10th, 18 & 0.48373 \\
\hline
\end{tabular}

Table 18: Discount curve on December 8th, 2003. 
- December 10th, 2003.

\begin{tabular}{|c|c||c|c||c|c|}
\hline Date & Discount & Date & Discount & Date & Discount \\
\hline Dec 10th, 03 & 1.00000 & Sep 13th, 04 & 0.98268 & Dec 12th, 11 & 0.71165 \\
Dec 11th, 03 & 0.99994 & Dec 13th, 04 & 0.97610 & Dec 12th, 12 & 0.67476 \\
Dec 15th, 03 & 0.99972 & Dec 12th, 05 & 0.94476 & Dec 12th, 13 & 0.63957 \\
Dec 19th, 03 & 0.99949 & Dec 12th, 06 & 0.90802 & Dec 12th, 14 & 0.60571 \\
Jan 12th, 04 & 0.99805 & Dec 12th, 07 & 0.86889 & Dec 14th, 15 & 0.57303 \\
Feb 12th, 04 & 0.99621 & Dec 12th, 08 & 0.82922 & Dec 12th, 16 & 0.54191 \\
Mar 12th, 04 & 0.99448 & Dec 14th, 09 & 0.78919 & Dec 12th, 17 & 0.51196 \\
Jun 14th, 04 & 0.98874 & Dec 13th, 10 & 0.74987 & Dec 12th, 18 & 0.48324 \\
\hline
\end{tabular}

Table 19: Discount curve on December 10th, 2003.

- March 10th, 2004.

\begin{tabular}{|c|c||c|c||c|c|}
\hline Date & Discount & Date & Discount & Date & Discount \\
\hline Mar 10th, 04 & 1.00000 & Dec 13th, 04 & 0.98422 & Mar 12th, 12 & 0.73537 \\
Mar 11th, 04 & 0.99994 & Mar 14th, 05 & 0.97884 & Mar 12th, 13 & 0.69831 \\
Mar 15th, 04 & 0.99972 & Mar 13th, 06 & 0.95375 & Mar 12th, 14 & 0.66271 \\
Mar 19th, 04 & 0.99949 & Mar 12th, 07 & 0.92279 & Mar 12th, 15 & 0.62817 \\
Apr 13th, 04 & 0.99807 & Mar 12th, 08 & 0.88770 & Mar 14th, 16 & 0.59552 \\
May 12th, 04 & 0.99642 & Mar 12th, 09 & 0.85063 & Mar 13th, 17 & 0.56392 \\
Jun 14th, 04 & 0.99455 & Mar 12th, 10 & 0.81234 & Mar 12th, 18 & 0.53344 \\
Sep 13th, 04 & 0.98940 & Mar 14th, 11 & 0.77355 & Mar 12th, 19 & 0.50440 \\
\hline
\end{tabular}

Table 20: Discount curve on March 10th, 2004.

\section{References}

[1] Bielecki T., Rutkowski M. (2001): Credit risk: Modeling, Valuation and Hedging. Springer Verlag.

[2] Black, F., and Cox, J.C. (1976). Valuing corporate securities: Some effects of bond indenture provisions. J. of Finance 31, 351-367.

[3] Brigo, D. (2004). Candidate Market Models and the Calibrated CIR++ Stochastic Intensity Model for Credit Default Swap Options and Callable Floaters. In Proceedings of the 4-th ICS Conference, Tokyo, March 18-19, 2004. Available at www.damianobrigo.it. Short version in "Market Models for CDS options and callable floaters", Risk Magazine, January 2005.

[4] Brigo, D. (2004b). Constant Maturity Credit Default Swap Pricing with Market Models. Available at ssrn.com. 
[5] Brigo, D., Alfonsi, A. (2005) Credit Default Swap Calibration and Derivatives Pricing with the SSRD Stochastic Intensity Model, Finance and Stochastics, Vol 9 (1), 2005. Extended verison available at www.damianobrigo.it. See also Proceedings of the 6-th Columbia=JAFEE International Conference, Tokyo, March 15-16, 2003, pages 563-585.

[6] Brigo D., Mercurio F. (2001): Interest Rate Models: Theory and Practice. Springer Verlag.

[7] Brigo D., and Tarenghi, M. (2005). Credit Default Swap Calibration and Counterparty Risk Pricing with a Scenario Volatility and Default Barrier Structural Model, presented at the Hybrid Equity/Credit WBS Workshop, London, March 15, 2005, forthcoming at ssrn.com and damianobrigo.it

[8] Crouhy, M., Galai, D., and Mark, R. (2000). A comparative analysis of current credit risk models. Journal of Banking and Finance 24, 59-117.

[9] Duffie D., Singleton K. (1999), Modeling term structures of defaultable bonds. Review of Financial Studies, 12, 687-720.

[10] Giesecke K. (2002). Correlated default with incomplete information. Cornell University working paper, to appear in J. of Banking and Finance.

[11] Lando, D. (1998), On Cox processes and credit-risky securities. Rev. Derivatives Res. 2, 99-120.

[12] Lo C. F., Lee H. C., Hui C. H. (2003): A Simple Approach for Pricing Barrier Options with Time-dependent Parameters. Quant. Fin. 3.

[13] Merton, R. (1974). On the pricing of corporate debt: The risk structure of interest rates. J. of Finance 29, 449-470.

[14] Metwally S. A. K., Atiya A. F. (2002): Using Brownian Bridge for Fast Simulation of Jump-Diffusion Processes and Barrier Options. The Journal of Derivatives.

[15] Pignatelli M. (2004): Private Communication, Banca IMI.

[16] Rapisarda F. (2003): Pricing Barriers on Underlyings with Time-dependent Parameters. Working Paper.

[17] Schönbucher P. (2003): Credit Derivatives Pricing Models: Model, Pricing and Implementation. Wiley. 